\title{
Applications of social constructivist learning theories in knowledge translation for healthcare professionals: a scoping review
}

\author{
Aliki Thomas ${ }^{1,2,3^{*}}$, Anita Menon', Jill Boruff ${ }^{4}$, Ana Maria Rodriguez ${ }^{1}$ and Sara Ahmed ${ }^{1,2}$
}

\begin{abstract}
Background: Use of theory is essential for advancing the science of knowledge translation (KT) and for increasing the likelihood that KT interventions will be successful in reducing existing research-practice gaps in health care. As a sociological theory of knowledge, social constructivist theory may be useful for informing the design and evaluation of KT interventions. As such, this scoping review explored the extent to which social constructivist theory has been applied in the KT literature for healthcare professionals.

Methods: Searches were conducted in six databases: Ovid MEDLINE (1948 - May 16, 2011), Ovid EMBASE, CINAHL, ERIC, PsycInfo, and AMED. Inclusion criteria were: publications from all health professions, research methodologies, as well as conceptual and theoretical papers related to KT. To be included in the review, key words such as constructivism, social constructivism, or social constructivist theories had to be included within the title or abstract. Papers that discussed the use of social constructivist theories in the context of undergraduate learning in academic settings were excluded from the review. An analytical framework of quantitative (numerical) and thematic analysis was used to examine and combine study findings.
\end{abstract}

Results: Of the 514 articles screened, 35 papers published between 1992 and 2011 were deemed eligible and included in the review. This review indicated that use of social constructivist theory in the KT literature was limited and haphazard. The lack of justification for the use of theory continues to represent a shortcoming of the papers reviewed. Potential applications and relevance of social constructivist theory in KT in general and in the specific studies were not made explicit in most papers. For the acquisition, expression and application of knowledge in practice, there was emphasis on how the social constructivist theory supports clinicians in expressing this knowledge in their professional interactions.

Conclusions: This scoping review was the first to examine use of social constructivism in KT studies. While the links between social constructivism and KT have not been fully explored, the Knowledge to Action framework has strong constructivist underpinnings that can be used in moving forward within the broader KT enterprise.

\section{Introduction}

Third party payers, insurers, professional regulatory boards, and patients increasingly expect healthcare professionals to integrate new knowledge and scientific evidence into daily practice $[1,2]$, with the ultimate goal of increasing their use of evidence-based practice (EBP) [3].

\footnotetext{
*Correspondence: aliki.thomas@mcgill.ca

${ }^{1}$ School of Physical and Occupational Therapy, McGill University, Montreal,

Quebec, Canada

${ }^{2}$ Centre for Interdisciplinary Rehabilitation Research of Greater Montreal,

Montreal, Quebec, Canada

Full list of author information is available at the end of the article
}

EBP has been shown to have a direct impact on improving patient outcomes [4].

Despite clear advantages for adhering to EBP principles, not all health professionals readily integrate scientific evidence into clinical decision making [5]. In the Netherlands and the United States, it is estimated that $30 \%$ to $45 \%$ of patients are not receiving care according to scientific evidence, and that $20 \%$ to $25 \%$ of the care provided is often unnecessary or potentially harmful $[6,7]$. In Canada, research studies in stroke rehabilitation have indicated that clinicians fail to routinely apply best practices [8-10]. For example, in a multi-center study of 
stroke rehabilitation therapists, Menon, Korner-Bitensky and Ogourtsova [11] found that only $13 \%$ of patients with unilateral spatial neglect (USN) post-stroke were assessed or screened with a standardized USN-specific tool during their acute care admission.

Recognition of the gap between what is known to improve patient outcomes and what is used in daily practice has led to a growing interest in knowledge translation (KT), defined as the exchange, synthesis and ethically sound application of knowledge to improve health and provide more effective health services [12]. Developing effective KT interventions that maximize clinicians' knowledge about best practices is an important step towards closing this knowledge-to-practice gap.

Some have argued that the use of theory is essential for advancing the science of $\mathrm{KT}$ and for increasing the likelihood of successful KT interventions for reducing these practice gaps [13-15]. Indeed, this is similar to the Medical Research Council's framework for the design of complex interventions, which stresses the importance of theory as a central part of designing, and testing interventions [83]. Greater use of theory can lead to a greater understanding of barriers and enablers of behavior change, inform the design of KT interventions, and allow for exploration of causal pathways and moderators for successful application of EBP [15]. Eccles et al. [14] highlighted how theories can be used to help design KT interventions and understand their impact on individuals and team behaviors. They emphasized that two objectives should be considered for the application of theories. The first objective is 'to develop an understanding of the theory-based factors that underlie clinical practice and to identify theoretical constructs that are important for current patterns of carethese should be the targets of a KT intervention' (p.3). This implies that theories could shed light on the multiple variables (both individual and organizational) that influence clinical behaviors, so that appropriate and targeted interventions can be designed to influence the likelihood that a given stakeholder will adopt a desired behavior. The second objective is 'to develop/test KT interventions that target specific theoretical constructs and to design these interventions for enhancing the processes that support change in them' [14] (p.3). While Eccles et al. (2005) and others $[16,17]$ recommend a more systematic use of theory to increase the chances of successful implementation, theories have been rarely used to inform the design and evaluation of KT interventions [5,18]. This observation was recently corroborated by Colquhoun et al. [19] and Davies, Walker, and Grimshaw [20] who also reported a limited use of KT theories, along with broader paradigms such as social cognitive theory, learning theories, and organizational theories. Colquhoun et al. [19] indicated that theories in KT studies tend to be mostly used in the fields of medicine and nursing, mainly to predict the success of KT interventions. A review by Davies et al. [20] found that only 6\% of included studies used theory to inform the design and/or the implementation of KT interventions. Most were theories of behavior or behavior change, including: 'diffusion of innovation', 'the theory of reasoned action', 'health beliefs model', and 'organizational development'. The review identified a number of studies reporting on KT interventions underpinned by two broad categories of theories: cognitive theories (e.g., social cognitive theory) and theories of learning (e.g., social learning theory). None of the studies reviewed were grounded in social constructivist theory [20].

\section{Potential application of social constructivist theories in KT} Several authors conceptualize KT as a process that occurs through social and environmental interactions, and emphasize that knowledge exchange between researchers and healthcare professionals must happen in a mutually created social context [21,31-33]. Indeed, knowledge use within $\mathrm{KT}$ can be regarded as an active learning process, because knowledge is not an inert object to be 'sent' and 'received', but a fluid set of understandings shaped by those who produce it and those who use it. Clinicians act upon new knowledge by transforming the information based on pre-existing experiences and understandings, by relating it to existing knowledge, imposing meaning to it and, in many cases, monitoring their understanding throughout the process. Hence, the meaning of research is constructed by the user and casts the clinician as an active problem solver and a constructor of his or her own knowledge, rather than a passive receptacle of information [22]. This has led us to propose that social constructivist theory may be useful for understanding why and how individuals integrate and apply new knowledge in evidence-based clinical decision making and how practice behaviors may change as a result of KT interventions grounded in the core tenets of this theory. We wish to emphasize that in this paper, we are focusing on constructivism, not constructionism. Though the two terms tend to be used interchangeably and often unapologetically [84], p.30, they are not synonyms. Social constructionism emphasizes purposeful creation of knowledge. The focus is on revealing the ways in which individuals and groups participate in the creation of their perceived social reality. It involves looking at the ways social phenomena are created, institutionalized and made into tradition by humans. Socially constructed reality is seen as an ongoing, dynamic process, and reality is reproduced by individuals acting on their interpretation and their knowledge. According to Burr (2003) there is no one feature which could be said to identify a social constructionist position, but there are assumptions among individuals who identify as such namely, 'a critical stance towards taken-for granted knowledge, historical and cultural 
specificity, knowledge is sustained by social processes and knowledge and social action go together' [85]. The social context is at the center of 'meaning making' in social constructionism and the attention is on the 'knowing' that is created through shared production. Constructionism also 'emphasizes the hold our culture has on us: it shapes the way in which we see things and gives us a quite definitive view of the world' [86] (p.58). In contrast, within a social constructivist paradigm, the individual is at the center of the meaning making experience. The focus of constructivism is on the individual's learning that takes place because of their interactions within a particular social context. According to Crotty (1998), 'it would appear useful, then, to reserve the term constructivism for epistemological considerations focusing exclusively on the meaning making activity of the individual mind' and to use constructionism where the focus include the collective generation [and transmission] of meaning' [86] (p.58). We privileged social constructivism as the focus of this review, for its emphasis on the individual and how/she he creates knowledge in socially medicated contexts.

Social constructivism is a sociological theory of knowledge that focuses on how individuals come to construct and apply knowledge in socially mediated contexts [21,22]. The fundamental premise of this theory is that knowledge is a human construction and that the learner is an active participant in the learning process [23]. Constructivism is based on three assumptions about learning [24-28]. First, learning is a result of the individual's interaction with the environment. Knowledge is constructed as the learner makes sense of their experiences in the world. The content of learning is not independent of how the learning is acquired; what a learner comes to understand is a function of the context of learning, the goals of the learner, and the activity the learner is involved in. Second, cognitive dissonance, or the uncomfortable tension that comes from holding two conflicting thoughts at the same time, is the stimulus for learning. It serves as a driving force that compels the mind to acquire new thoughts or to modify existing beliefs in order to reduce the amount of dissonance (conflict). Cognitive dissonance ultimately determines the organization and nature of what is learned [29]. Third, the social environment plays a critical role in the development of knowledge. Other individuals in the environment may attempt to test the learner's understanding and provide alternative views against which the learner questions the viability of his knowledge. Constructivism supports the acquisition of cognitive processing strategies, self-regulation, and problem solving through socially constructed learning opportunities $[25,26,28,30]$, all of which are critical skills for evidencebased knowledge uptake and implementation in clinical practice [31].
The Knowledge to Action (KTA) framework [32] adopted by the Canadian Institutes for Health Research, is a widely used framework that focuses on knowledge creation and exchange. The KTA framework contains two principal components, a knowledge creation funnel and an action cycle. The knowledge creation funnel consists of three phases: knowledge inquiry, knowledge synthesis, and knowledge tools and products. The action cycle consists of seven stages involved in moving knowledge into practice: identifying a problem in practice or a gap in knowledge and identifying, reviewing, and selecting the knowledge to be implemented to address the gap; adapting or customizing the knowledge to the local context; evaluating the determinants of the knowledge use (barriers and facilitators); selecting, tailoring and implementing interventions to address the knowledge or practice gap; monitoring the knowledge use in practice; evaluating the outcomes or impact of using the new knowledge; and determining strategies for ensuring that the new knowledge is sustained [32]. The KTA framework is grounded in the social constructivist paradigm which privileges social interaction and adaptation of research evidence by taking the local context and culture into account [34]. To our knowledge, this is the only KT framework developed with social constructivist underpinnings. Despite the growing recognition that the KTA framework can facilitate knowledge use and exchange in practice, its association with social constructivist theory has yet to be explicitly explored.

Social constructivist approaches to the science of KT have the potential to support researchers interested in examining how learning in the clinical context occurs and how new knowledge is created, disseminated, exchanged and used to inform practice. While social constructivist theory may be useful for informing the design and evaluation of KT interventions, we have yet to understand the extent to which social constructivist theory has been applied in the KT literature for healthcare professionals. Thus, this paper presents the results of a scoping review on the application of social constructivist theory in KT for healthcare professionals.

\section{Methods}

There are four reasons for undertaking scoping reviews: to examine the extent, range and nature of research activity, to determine the value of undertaking a systematic review, to summarize and disseminate research findings, and to identify research gaps in the existing literature [35]. The objectives of the scoping review reported in this paper were to summarize and disseminate findings from a broad body of literature and identify research gaps in the existing literature. Using the Arksey and O'Malley framework [35], we outline the specific methods for our scoping review below: 


\section{Step one: research question}

The research question that guided the review was 'What are the applications of social constructivism and/or social constructivist theories in KT to promote EBP among healthcare professionals'? We used the PICOS format as a structure for our research question and to design our search strategy. The population is 'healthcare professionals'; intervention is 'application of social constructivism in KT'; the outcome is 'promote EBP'; and the study design refers to all the study designs eligible for inclusion in the review. Eligible study designs included: all qualitative methodologies and quantitative designs (observations studies, randomized controlled trials, cohort studies, cross sectional studies, longitudinal studies and case studies).

Step two: identifying relevant studies and study selection All members of the research team were involved in decisions about inclusion and exclusion criteria. The team worked with a rehabilitation sciences librarian (JB) who suggested that, given our research question, we take a broad approach to the concept of KT when selecting search terms. The terms captured both the theory and application of KT as discussed by McKibbon [36], and took into account the terms used by a previous systematic review on $\mathrm{KT}$ in rehabilitation [37]. A first pilot search was constructed to include articles where any variation of the word 'constructivism' or 'constructivist' appeared in the title or abstract of articles discussing health professionals. A research assistant under the supervision of one member of the research team (AT) was responsible for reading the abstracts of all the articles identified in this first search and applying the original inclusion/ exclusion criteria in an abstract screening tool. Two members of the research team (AT and AM) piloted the inclusion/exclusion criteria with a subset of abstracts retrieved from MEDLINE. The same two members of the research team (AT and AM) reviewed the search terms, the redesigned strategy and approved the abstract screening tool. This process resulted in modifications to the inclusion/exclusion criteria and the search was redesigned to include: publications from all health professions; all research methodologies (quantitative and qualitative); conceptual and theoretical papers related to $\mathrm{KT}$; and, papers written in English. Excluded from the review were papers that had no evidence of the concept of knowledge translation in the abstract; were unrelated to any health profession or health field; discussed new curricula designed to promote higher level learning in health sciences students; and described new pedagogical methods (i.e., virtual, simulating techniques, etc.) for teaching in schools.

The pilot search developed for MEDLINE was conducted again with the new inclusion criteria, and then adapted for other databases. The Ovid MEDLINE search strategy is presented as 'Additional file 1'. Searches were conducted in six databases: Ovid MEDLINE (1948 - May 16, 2011), Ovid EMBASE (1980 - May 16, 2011), CINAHL (searched entire database to May 16, 2011), ERIC (1966 May 16, 2011), PsycInfo (1967 - May 16, 2011), and AMED (1985 - May 16, 2011). The librarian used GoPubMed to analyze the subject headings of the fulltext articles that were assessed and considered for eligibility (see PRISMA flow chart) and then again of the final articles to determine whether any important terms had been missed. The iterative nature of scoping reviews allowed the research team to consider the addition of articles that best reflected new ideas gained from the review process. The GoPubMed analysis added seven other medical subject headings to the search (line 21 in Additional file 1). An expanded search including these new subject headings was conducted six months later.

\section{Step three: charting the data}

The authors developed a data charting form that included the following categories: author, year of publication, purpose of the study/research question, practice setting, nature of theory use, links with the KTA framework, methodology, population characteristics, outcome evaluation (evaluation setting, evaluation responses, effectiveness of implementation, variables of evaluation, outcomes), implications for practice, and directions for future research. The data charting form was piloted on the first 10 articles and reviewed by the research team to ensure that it was comprehensive. A research assistant extracted the data for the remaining articles. The two senior authors (AT and SA) reviewed and discussed the completed extraction tables. Categories such as 'population characteristics', 'outcome evaluation', 'effectiveness of implementation', and 'evaluation variables' were not appropriate for several conceptual papers and were adapted to be more inclusive. This was an iterative process that ensured that the tables included all the salient information for generating the themes as per step four described below.

\section{Step four: collating, summarizing and reporting the results}

An analytical framework of quantitative (numerical) and thematic analysis was used to examine and combine study findings [35]. The numerical analysis highlighted: the nature and distribution of the studies; the nature of the social constructivist assumptions used in each study; and the KTA stage/component targeted.

The nature of the application or use of social constructivism across all papers served as the major unit of analysis. We also aimed to identify which of the three social constructivist assumptions were used in the selected studies. Two members of the research team (AT and SA) independently reviewed the data charting tables and identified a number of preliminary 
emerging themes. All other members of the research team were consulted to discuss the themes and ensure agreement. This process resulted in the generation of five themes. With the assistance of a doctoral student (AMR), we revisited all the charting tables to confirm that these corresponded with the themes that were generated. A summary of the major findings organized under each theme was produced following several iterations and meetings with the research team.

\section{Results}

\section{Nature and distribution of the studies}

A total of 855 results were retrieved from all sources. Duplicates were removed $(\mathrm{n}=341)$, yielding 514 records for eligibility screening. We screened the 514 abstracts and excluded 437 papers on the basis of our four exclusion criteria. Seventy-seven articles were read in full and assessed for eligibility. Fourty-two additional papers were excluded for the following reasons: no evidence of the concept of knowledge translation in the abstract $(\mathrm{n}=8)$; study was unrelated to any health profession or health field $(n=8)$; study findings were related to new online curriculum designs in higher level learning for health sciences students $(\mathrm{n}=10)$; study was based on new pedagogical methods (i.e., virtual, simulating techniques, etc.) for teaching in schools or was conducted in an educational setting with undergraduate students in health sciences $(n=16)$. The number of eligible article at this stage was 35 . The expanded search resulted in an additional 55 additional articles plus seven MEDLINE articles for screening for a total of 62 additional articles for screening. In the end however, none of these new articles from the expanded search were eligible for the final review. The numbers of articles at each stage selection process are shown in the PRISMA flow chart (Figure 1).

Thirty-five papers published between 1992 and 2011 met the inclusion criteria. Table 1 shows the charting categories and associated content for the 35 studies. Tables 2, 3, 4 represent the study designs, practice setting, and professional groups respectively. Twenty-seven studies used a qualitative study design. These ranged from various types of literature reviews, conceptual and reflective papers to studies using interviews and questionnaires, focus groups and observations. Six papers described the results of KT interventions that for the most part, consisted of a workshop or a didactic course [38-43]. Two studies used a mixed method design $[44,45]$ (Table 2). The most common practice settings identified were primary healthcare $(\mathrm{n}=10)$ $[42,44,53,59-62,64,65,82]$, followed by post-graduate educational settings $(\mathrm{n}=7)[39-41,43,45,65,81]$, and mental health clinical environments $(n=5)[50,52,58,78,80]$ (Table 3$)$. Nursing was the professional group most frequently targeted in the papers (20 of 35 included studies), alone $[42,43,47,53,55,60-62,77]$, or along with physicians [64,82], patients [44], or interdisciplinary teams $[38,39,48,51,57,65,76,79]$. Psychologists/psychiatrists was another identified group $(n=5)[50,52,58,78,80]$. Four papers $[40,41,45,80]$ presented results of studies conducted with postgraduate (e.g., residents and other trainees not considered undergraduate learners) health care professionals (Table 4).

\section{Social constructivist assumptions}

Table 5 illustrates that 15 papers discussed research grounded in the social constructivist assumption 'learning is a result of the individual's interaction with the environment'. Eight studies corresponded to the assumption that 'the social environment plays a critical role in the development of knowledge' and four studies were about 'cognitive dissonance as the stimulus for learning'. Eight studies explored all three assumptions.

\section{Stages of the knowledge-to-action cycle}

As shown in Table 5, 13 studies involved knowledge creation ( $\mathrm{n}=7$ knowledge synthesis, $\mathrm{n}=5$ knowledge inquiry and $\mathrm{n}=1$ knowledge tools). Twenty-two studies addressed one of the four specific steps of the action cycle: four studies addressed step one, 'identify the problem or knowledge gap', two studies addressed 'adapting knowledge to local context' (step two), and the remaining were equally divided between step three 'assessing barriers and facilitators' $(\mathrm{n}=8)$ and step four 'select, tailor and implement intervention' $(\mathrm{n}=8)$. No study mapped onto more than one step of the action cycle.

\section{Thematic analysis}

We identified five themes related to the applications of social constructivist theory in KT with several nested concepts within each theme (Table 6).

\section{Theme one: meaning of evidence and tension between research and practice ( $\mathrm{n}=9$ papers)}

The papers [46-52,76,79] in this theme reported findings from literature reviews exploring the meaning of evidence and the epistemology of research and practice. Papers in this theme recognized that there may be various definitions of 'knowledge' and 'knowledge creation' which may vary depending upon the theoretical lens used to explore the applications of knowledge in clinical practice. Adler's [46] review of the history of science literature suggested that different types of evidence can and should be used in health research. Appleton [47] discussed the relevance of constructivism to researchers in health services while Labonte's [48] literature review on the social constructivist paradigm in health promotion research, suggested that this paradigm has the potential to resolve some of the philosophical tensions between research and practice in health promotion. 


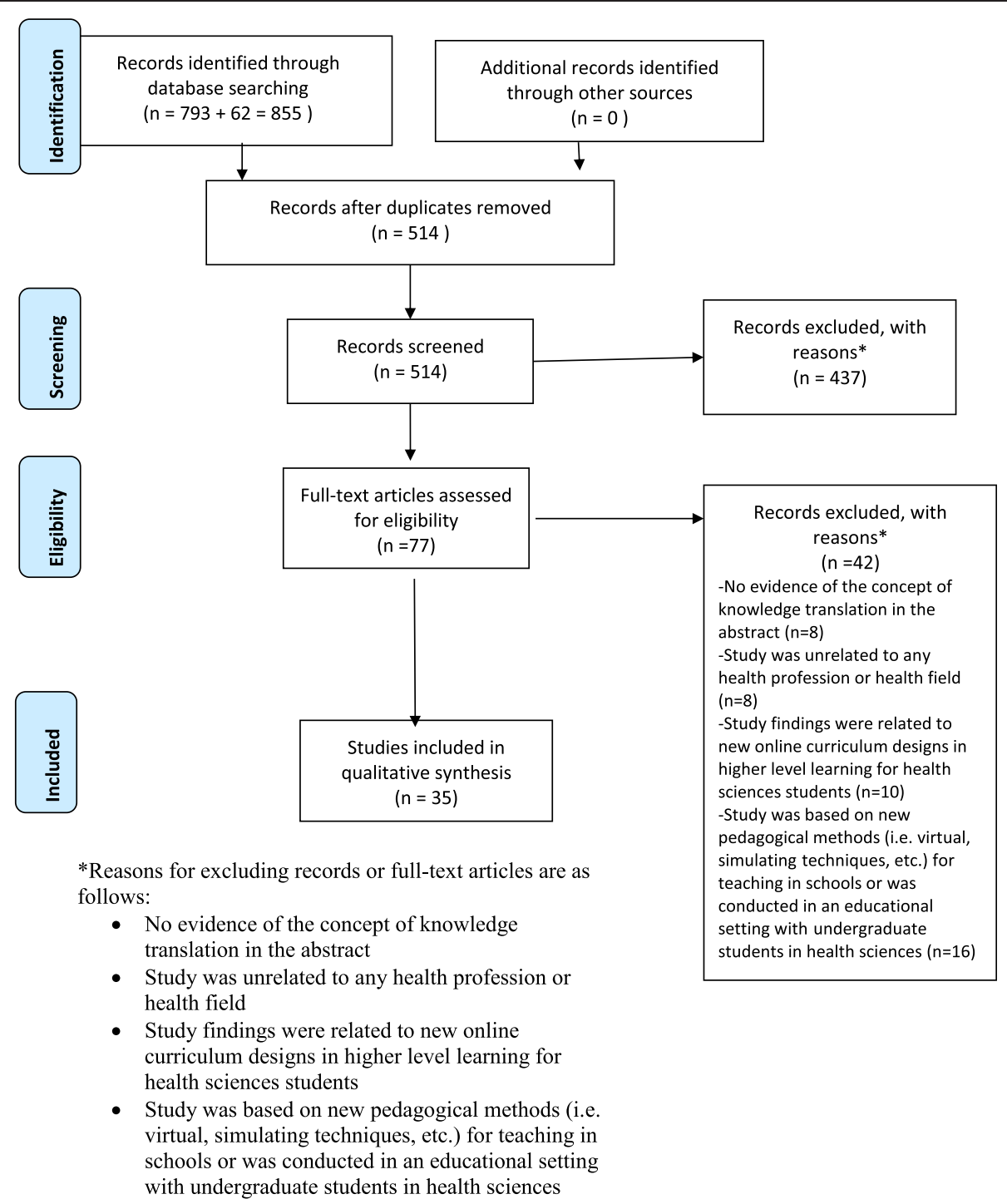

Figure 1 PRISMA Flow Diagram. *Reasons for excluding records or full-text articles are as follows: no evidence of the concept of knowledge translation in the abstract; study was unrelated to any health profession or health field; study findings were related to new online curriculum designs in higher level learning for health sciences students; study was based on new pedagogical methods (i.e., virtual, simulating techniques, etc.) for teaching in schools or was conducted in an educational setting with undergraduate students in health sciences.

Plack [49] suggested that physical therapists should shift their focus from mainly positivist approaches to care to more constructivist ones in order that they may make better use of evidence. In another literature review, Miller [50] found that the social constructivist paradigm could serve as a bridge between researchers and practitioners by suggesting that research efforts be directed towards identifying the needs of those who will be offering and receiving health care services. Wilson [51] discussed the biomedical model of care and introduced a debate on the effectiveness of objectivism in health care. The authors suggested that a more subjectivist model to healthcare, and one that embraces social constructivist theories, would include recent evidence on doctor-patient relationship as a major contributor to patient outcomes in addition to incorporating 'objective' clinical findings. Hoshmand's [52] literature review emphasized a broader choice of research methods, the development of reflective skills in practice and better linkages between researchers and practitioners.

Theme two: understanding of acquisition, expression and application of knowledge in and for professional practice ( $n=14$ papers)

Social constructivism was used as a lens through which to gain a greater understanding of how knowledge is acquired, manifested and used to inform practice as well as to explain the individual and contextual factors that have an impact on skill development and/or behavior 
Table 1 Descriptive information for each study included in the scoping review

\begin{tabular}{|c|c|c|c|c|c|c|c|c|}
\hline $\begin{array}{l}\text { First author } \\
\text { (Year) }\end{array}$ & Main theme & Area of practice & Target population & $\begin{array}{l}\text { Study } \\
\text { design }\end{array}$ & $\begin{array}{l}\text { Intervention/ } \\
\text { approach }\end{array}$ & Main findings & $\begin{array}{l}\text { Theory } \\
\text { described }\end{array}$ & $\begin{array}{l}\text { Theory } \\
\text { integrated }\end{array}$ \\
\hline \multirow{3}{*}{$\begin{array}{l}\text { Abad-Corpa } \\
\text { (2010) }[44]\end{array}$} & \multirow{3}{*}{$\begin{array}{l}\text { Design of a KT } \\
\text { activity/intervention } \\
\text { (to improve EBP in } \\
\text { nurses and } \\
\text { outcomes in patient) }\end{array}$} & \multirow{3}{*}{$\begin{array}{l}\text { Primary health care } \\
\text { setting }\end{array}$} & - Nurses & \multirow{3}{*}{$\begin{array}{l}\text { Mixed } \\
\text { (qualitative } \\
\text { approach, } \\
\text { quantitative } \\
\text { analysis) }\end{array}$} & \multirow{3}{*}{$\begin{array}{l}\text { Focus groups } \\
\text { (reviewed articles, } \\
\text { videos, field diaries, } \\
\text { statistics) }\end{array}$} & -Psycho-social adjustment & \multirow[t]{3}{*}{ Yes } & \multirow[t]{3}{*}{ yes } \\
\hline & & & \multirow{2}{*}{$\begin{array}{l}\text { - Patients (with } \\
\text { compromised immune } \\
\text { system) }\end{array}$} & & & -Satisfaction with nursing & & \\
\hline & & & & & & -Family burden & & \\
\hline $\begin{array}{l}\text { Adler (2002) } \\
\text { [46] }\end{array}$ & $\begin{array}{l}\text { Meaning of } \\
\text { 'evidence', tension } \\
\text { between research } \\
\text { and practice }\end{array}$ & $\mathrm{N} / \mathrm{A}$ & $\begin{array}{l}\text { Focus on Physicians } \\
\text { involved in research }\end{array}$ & Qualitative & $\begin{array}{l}\text { Review of the } \\
\text { literature and history } \\
\text { of science }\end{array}$ & $\begin{array}{l}\text { Reflection on the type of evidences to use } \\
\text { in health research }\end{array}$ & Yes & N/A \\
\hline $\begin{array}{l}\text { Appleton } \\
\text { (2002) [47] }\end{array}$ & $\begin{array}{l}\text { Meaning of } \\
\text { 'evidence', tension } \\
\text { between research } \\
\text { and practice }\end{array}$ & N/A & $\begin{array}{l}\text { Focus on Health services } \\
\text { researchers }\end{array}$ & Qualitative & $\begin{array}{l}\text { Review of the types of } \\
\text { philosophical } \\
\text { approaches and } \\
\text { reflection on the } \\
\text { implication for } \\
\text { practice }\end{array}$ & $\begin{array}{l}\text { Philosophical underpinnings of } \\
\text { constructivism and relevance to } \\
\text { researchers in health services }\end{array}$ & Yes & $\begin{array}{l}\text { N/A but } \\
\text { links with } \\
\text { health } \\
\text { research } \\
\text { emphasized }\end{array}$ \\
\hline $\begin{array}{l}\text { Carr (2005) } \\
{[54]}\end{array}$ & $\begin{array}{l}\text { Acquisition, } \\
\text { expression and } \\
\text { application of } \\
\text { knowledge for } \\
\text { professional practice }\end{array}$ & $\mathrm{N} / \mathrm{A}$ & Focus on nurses & Qualitative & $\begin{array}{l}\text { Reflective/guidance } \\
\text { elaboration }\end{array}$ & $\begin{array}{l}\text { The interpretive paradigm provides one } \\
\text { means of voicing nursing knowledge. }\end{array}$ & Briefly & N/A \\
\hline $\begin{array}{l}\text { Caley (2010) } \\
{[38]}\end{array}$ & $\begin{array}{l}\text { Design of a } \mathrm{KT} \\
\text { activity/intervention }\end{array}$ & $\begin{array}{l}\text { Health and human } \\
\text { services } \\
\text { organization }\end{array}$ & $\begin{array}{l}\text { Health and Human service } \\
\text { professionals }\end{array}$ & Intervention & $\begin{array}{l}\text { Workshop on alcohol } \\
\text { dependence } \\
\text { screening, survey }\end{array}$ & Number of interventions implemented & Yes & Yes \\
\hline $\begin{array}{l}\text { Cronin } \\
(2007)[45]\end{array}$ & $\begin{array}{l}\text { Design of a } \mathrm{KT} \\
\text { activity/intervention }\end{array}$ & $\begin{array}{l}\text { Education (post- } \\
\text { graduate training of } \\
\text { health care } \\
\text { professionals) }\end{array}$ & $\begin{array}{l}\text { Post-graduate health } \\
\text { promotion education }\end{array}$ & Intervention & $\begin{array}{l}\text { Workshop on } \\
\text { experiential learning, } \\
\text { reflective practice, } \\
\text { satisfaction survey }\end{array}$ & Student satisfaction & $\begin{array}{l}\text { Yes in } \\
\text { part }\end{array}$ & Yes \\
\hline $\begin{array}{l}\text { Daley } \\
\text { (2001) [39] }\end{array}$ & $\begin{array}{l}\text { Acquisition, } \\
\text { expression and } \\
\text { application of } \\
\text { knowledge for } \\
\text { professional practice }\end{array}$ & $\begin{array}{l}\text { Education (post- } \\
\text { graduate training of } \\
\text { health care } \\
\text { professionals) }\end{array}$ & $\begin{array}{l}\text { Social workers, lawyers, } \\
\text { nurses, educators }\end{array}$ & Intervention & $\begin{array}{l}\text { Post-graduate course } \\
\text { followed by survey }\end{array}$ & $\begin{array}{l}\text { Identification of key components that } \\
\text { made knowledge useful }\end{array}$ & Yes & Yes \\
\hline $\begin{array}{l}\text { Fagan (1998) } \\
{[62]}\end{array}$ & $\begin{array}{l}\text { To better understand } \\
\text { clients, and their } \\
\text { experiences/realities }\end{array}$ & $\begin{array}{l}\text { Primary health care } \\
\text { setting }\end{array}$ & Emergency nurses & Qualitative & Questionnaire & $\begin{array}{l}\text { Perception of nurses regarding their roles } \\
\text { in identifying child abuse }\end{array}$ & No & No \\
\hline $\begin{array}{l}\text { Fairweather } \\
\text { (2000) [61] }\end{array}$ & $\begin{array}{l}\text { Learning in } \\
\text { promoting } \\
\text { professional } \\
\text { expertise }\end{array}$ & $\begin{array}{l}\text { Primary health care } \\
\text { setting }\end{array}$ & Specialist nurses & Qualitative & Focus groups & $\begin{array}{l}\text { Roles and attributes of specialist vs } \\
\text { generalist nursing }\end{array}$ & Yes & Yes in part \\
\hline $\begin{array}{l}\text { Felton } \\
\text { (2003) [76] }\end{array}$ & $\begin{array}{l}\text { Meaning of } \\
\text { 'evidence', tension } \\
\text { between research } \\
\text { and practice }\end{array}$ & Community services & $\begin{array}{l}\text { Mental health, social } \\
\text { services, community } \\
\text { services, hospital } \\
\text { administrators involved in }\end{array}$ & Qualitative & Interview & $\begin{array}{l}\text { Consensus on system-level concerns re- } \\
\text { garding involvement of outside agency in } \\
\text { 'Housing first' projects }\end{array}$ & Yes & Yes \\
\hline
\end{tabular}


Table 1 Descriptive information for each study included in the scoping review (Continued)

\begin{tabular}{|c|c|c|c|c|c|c|c|c|}
\hline & & & $\begin{array}{l}\text { shelters and housing } \\
\text { accessibility }\end{array}$ & & & & & \\
\hline $\begin{array}{l}\text { Field (2004) } \\
\text { [55] }\end{array}$ & $\begin{array}{l}\text { Acquisition, } \\
\text { expression and } \\
\text { application of } \\
\text { knowledge for } \\
\text { professional practice }\end{array}$ & N/A & Focus on Nurses & Qualitative & Literature review & $\begin{array}{l}\text { Importance of context in learning and } \\
\text { difficulty of transferring knowledge to } \\
\text { different context }\end{array}$ & Yes & N/A \\
\hline $\begin{array}{l}\text { Fonville } \\
\text { (2002) [53] }\end{array}$ & $\begin{array}{l}\text { Acquisition, } \\
\text { expression and } \\
\text { application of } \\
\text { knowledge for } \\
\text { professional practice }\end{array}$ & $\begin{array}{l}\text { Primary health care } \\
\text { setting }\end{array}$ & Nurse executives & Qualitative & Interview & $\begin{array}{l}\text { Nursing are more loyal to their professional } \\
\text { than their organizational entity, unaware of } \\
\text { ethics principles, need for reflective } \\
\text { learning. }\end{array}$ & $\begin{array}{l}\text { Yes in } \\
\text { part }\end{array}$ & Yes \\
\hline $\begin{array}{l}\text { Greenhalgh } \\
\text { (2006) [65] }\end{array}$ & $\begin{array}{l}\text { Design of a } \mathrm{KT} \\
\text { activity/intervention }\end{array}$ & $\begin{array}{l}\text { Education (post- } \\
\text { graduate training of } \\
\text { health care } \\
\text { professionals) }\end{array}$ & $\begin{array}{l}\text { Senior professionals: senior } \\
\text { partners in general practice, } \\
\text { postgraduate tutors, service } \\
\text { managers }\end{array}$ & $\begin{array}{l}\text { Online } \\
\text { course }\end{array}$ & $\begin{array}{l}\text { Student Course } \\
\text { Evaluation }\end{array}$ & $\begin{array}{l}\text { Web-based learning offers potential for } \\
\text { students to engage in rich and effective } \\
\text { construction of knowledge. }\end{array}$ & Briefly & No \\
\hline $\begin{array}{l}\text { Greenslade } \\
(2010)[63]\end{array}$ & $\begin{array}{l}\text { To better understand } \\
\text { clients, and their } \\
\text { experiences/realities }\end{array}$ & $\begin{array}{l}\text { Primary health care } \\
\text { setting (same-day } \\
\text { surgery) }\end{array}$ & $\begin{array}{l}\text { Breast cancer surgery } \\
\text { patients }\end{array}$ & Qualitative & Interview & $\begin{array}{l}\text { Follow-up visit for assessment, education, } \\
\text { and psychosocial support recommended. }\end{array}$ & No & No \\
\hline $\begin{array}{l}\text { Higgs } \\
\text { (1995) [56] }\end{array}$ & $\begin{array}{l}\text { Acquisition, } \\
\text { expression and } \\
\text { application of } \\
\text { knowledge for } \\
\text { professional practice }\end{array}$ & N/A & Focus on Physical Therapists & Qualitative & Literature review & $\begin{array}{l}\text { Knowledge is an active and dynamic } \\
\text { phenomenon undergoing constant } \\
\text { changes and testing }\end{array}$ & Yes & $\mathrm{N} / \mathrm{A}$ \\
\hline $\begin{array}{l}\text { Holtslander } \\
\text { (2008) [77] }\end{array}$ & $\begin{array}{l}\text { Acquisition, } \\
\text { expression and } \\
\text { application of } \\
\text { knowledge for } \\
\text { professional practice }\end{array}$ & N/A & Focus on palliative nurses & Qualitative & Reflective paper & $\begin{array}{l}\text { Exposition of the ways to acquire } \\
\text { knowledge and the nursing model in } \\
\text { palliative setting }\end{array}$ & Briefly & N/A \\
\hline $\begin{array}{l}\text { Hoshmand } \\
\text { (1992) [52] }\end{array}$ & $\begin{array}{l}\text { Meaning of } \\
\text { 'evidence', tension } \\
\text { between research } \\
\text { and practice }\end{array}$ & N/A & $\begin{array}{l}\text { Focus on psychological } \\
\text { Sciences }\end{array}$ & Qualitative & Literature review & $\begin{array}{l}\text { Emphasis on broadened choices of } \\
\text { research methods, the development of } \\
\text { reflective skills, and better linkage between } \\
\text { teaching in the domains of research and } \\
\text { practice are urged. }\end{array}$ & yes & N/A \\
\hline $\begin{array}{l}\text { Hunter } \\
(2008)[40]\end{array}$ & $\begin{array}{l}\text { Design of a } K T \\
\text { activity/intervention }\end{array}$ & $\begin{array}{l}\text { Education (post- } \\
\text { graduate training of } \\
\text { health care } \\
\text { professionals) }\end{array}$ & Nurses & Intervention & $\begin{array}{l}\text { Course and Student } \\
\text { Course Evaluation and } \\
\text { students' cultural } \\
\text { competence levels } \\
\text { evaluations }\end{array}$ & $\begin{array}{l}\text { Students' comments were all positive or } \\
\text { politely constructive, their competency } \\
\text { increased. }\end{array}$ & Yes & Yes \\
\hline $\begin{array}{l}\text { Kinsella } \\
(2010)[57]\end{array}$ & $\begin{array}{l}\text { Acquisition, } \\
\text { expression and } \\
\text { application of } \\
\text { knowledge for } \\
\text { professional practice }\end{array}$ & N/A & $\begin{array}{l}\text { Focus on practice in } \\
\text { nursing, health and social } \\
\text { care professions }\end{array}$ & Qualitative & Reflective paper & $\begin{array}{l}\text { Discerning philosophical underpinnings of } \\
\text { reflective practice to advance increasingly } \\
\text { coherent interpretations }\end{array}$ & Yes & $\mathrm{N} / \mathrm{A}$ \\
\hline $\begin{array}{l}\text { Labonte } \\
\text { (1996) [48] }\end{array}$ & $\begin{array}{l}\text { Meaning of } \\
\text { 'evidence', tension }\end{array}$ & $\mathrm{N} / \mathrm{A}$ & Focus on health promotion & Qualitative & Literature review & $\begin{array}{l}\text { A 'constructivist' research paradigm has the } \\
\text { potential to resolve some of the tensions }\end{array}$ & Yes & $\mathrm{N} / \mathrm{A}$ \\
\hline
\end{tabular}


Table 1 Descriptive information for each study included in the scoping review (Continued)

\begin{tabular}{|c|c|c|c|c|c|c|c|c|}
\hline & $\begin{array}{l}\text { between research } \\
\text { and practice }\end{array}$ & & & & & $\begin{array}{l}\text { between research and practice in health } \\
\text { promotion }\end{array}$ & & \\
\hline $\begin{array}{l}\text { Lipman } \\
\text { (2005) [59] }\end{array}$ & $\begin{array}{l}\text { Acquisition, } \\
\text { expression and } \\
\text { application of } \\
\text { knowledge for } \\
\text { professional practice }\end{array}$ & $\begin{array}{l}\text { Primary health care } \\
\text { setting }\end{array}$ & $\begin{array}{l}\text { Physicians researchers in } \\
\text { anticoagulation in patients } \\
\text { with atrial fibrillation }\end{array}$ & Qualitative & Interviews & $\begin{array}{l}\text { Implementing research evidence is more } \\
\text { complex than in suggested in current } \\
\text { models of evidence-based medicine }\end{array}$ & Yes & No \\
\hline $\begin{array}{l}\text { Lyddon } \\
\text { (2006) [78] }\end{array}$ & $\begin{array}{l}\text { Acquisition, } \\
\text { expression and } \\
\text { application of } \\
\text { knowledge for } \\
\text { professional practice }\end{array}$ & $\begin{array}{l}\text { Focuses on } \\
\text { counselling (mental } \\
\text { health services) }\end{array}$ & Focus on Psychology & Qualitative & $\begin{array}{l}\text { Literature review / } \\
\text { reflection }\end{array}$ & $\begin{array}{l}\text { Emerging research strategy in self } \\
\text { confrontation method, proven to be a } \\
\text { useful procedure for practitioners in } \\
\text { counseling settings }\end{array}$ & Yes & Yes \\
\hline $\begin{array}{l}\text { McGuckin } \\
\text { (2006) [58] }\end{array}$ & $\begin{array}{l}\text { Acquisition, } \\
\text { expression and } \\
\text { application of } \\
\text { knowledge for } \\
\text { professional practice }\end{array}$ & $\begin{array}{l}\text { Not expressed, but } \\
\text { most probably } \\
\text { mental health } \\
\text { services since focus } \\
\text { is on psychiatry }\end{array}$ & Focus on Psychiatry & Qualitative & $\begin{array}{l}\text { Literature review / } \\
\text { reflection }\end{array}$ & $\begin{array}{l}\text { An eclectic approach that combines } \\
\text { elements of the directed approach and the } \\
\text { constructivist approach seems warranted }\end{array}$ & Yes & $\mathrm{N} / \mathrm{A}$ \\
\hline $\begin{array}{l}\text { McWilliam } \\
\text { (2009) [79] }\end{array}$ & $\begin{array}{l}\text { Meaning of } \\
\text { 'evidence', tension } \\
\text { between research } \\
\text { and practice }\end{array}$ & $\begin{array}{l}\text { Home care } \\
\text { programs }\end{array}$ & $\begin{array}{l}\text { Service providers, case } \\
\text { managers, administrators, } \\
\text { researchers }\end{array}$ & Qualitative & $\begin{array}{l}\text { Action groups to } \\
\text { implement KT through } \\
\text { social interaction }\end{array}$ & $\begin{array}{l}\text { Sharing accountability for implementation } \\
\text { is challenging for achievement-oriented re- } \\
\text { searchers and quality health care } \\
\text { practitioners }\end{array}$ & Yes & Yes \\
\hline $\begin{array}{l}\text { Miller } \\
(2002)[50]\end{array}$ & $\begin{array}{l}\text { Meaning of } \\
\text { 'evidence', tension } \\
\text { between research } \\
\text { and practice }\end{array}$ & $\begin{array}{l}\text { Not expressed, but } \\
\text { most probably } \\
\text { mental health } \\
\text { services since focus } \\
\text { is on psychiatry }\end{array}$ & $\begin{array}{l}\text { Focus on trauma- psychiatry } \\
\text { researchers }\end{array}$ & Qualitative & $\begin{array}{l}\text { Literature review / } \\
\text { reflection }\end{array}$ & $\begin{array}{l}\text { social constructivism can serve as a bridge } \\
\text { between researchers and practitioners by } \\
\text { refocusing research efforts to the needs of } \\
\text { war-affected communities }\end{array}$ & Yes & N/A \\
\hline $\begin{array}{l}\text { Neimeyer } \\
\text { (1998) [80] }\end{array}$ & $\begin{array}{l}\text { Acquisition, } \\
\text { expression and } \\
\text { application of } \\
\text { knowledge for } \\
\text { professional practice }\end{array}$ & $\begin{array}{l}\text { Mental health } \\
\text { services since focus } \\
\text { is on psychiatry }\end{array}$ & $\begin{array}{l}\text { Focus on Psychology- } \\
\text { counselling services }\end{array}$ & Qualitative & $\begin{array}{l}\text { Reflection on the } \\
\text { literature }\end{array}$ & $\begin{array}{l}\text { Discusses the theories of SC that may } \\
\text { support the importation of this theory into } \\
\text { the counselling context }\end{array}$ & Yes & N/A \\
\hline $\begin{array}{l}\text { Plack (2005) } \\
\text { [49] }\end{array}$ & $\begin{array}{l}\text { Meaning of } \\
\text { 'evidence', tension } \\
\text { between research } \\
\text { and practice }\end{array}$ & N/A & Focus on Physical Therapy & Qualitative & Literature review & $\begin{array}{l}\text { PT research should shift its focus from } \\
\text { mainly positivism to include constructivism } \\
\text { and critical theory for practitioners to } \\
\text { better use the evidence }\end{array}$ & Yes & $\mathrm{N} / \mathrm{A}$ \\
\hline $\begin{array}{l}\text { Rogal } \\
(2008)[41]\end{array}$ & $\begin{array}{l}\text { Design of a KT } \\
\text { activity/intervention }\end{array}$ & $\begin{array}{l}\text { Education (post- } \\
\text { graduate training of } \\
\text { nurses) }\end{array}$ & $\begin{array}{l}\text { Graduate nurses in a } \\
\text { Problem-based learning } \\
\text { session }\end{array}$ & Intervention & $\begin{array}{l}\text { Course and } \\
\text { Satisfaction about } \\
\text { education program }\end{array}$ & $\begin{array}{l}\text { Step-by-step guide of constructing a } \\
\text { problem based learning package for large, } \\
\text { single session groups }\end{array}$ & Yes & Yes \\
\hline $\begin{array}{l}\text { Rogers } \\
(2011)[64]\end{array}$ & $\begin{array}{l}\text { Design of a KT } \\
\text { activity/intervention }\end{array}$ & $\begin{array}{l}\text { Primary health care } \\
\text { setting }\end{array}$ & $\begin{array}{l}\text { Surgeons and Nurses in OR } \\
\text { teams }\end{array}$ & Qualitative & $\begin{array}{l}\text { Focus groups on team } \\
\text { conflict }\end{array}$ & $\begin{array}{l}\text { Source of conflict are mainly task-related } \\
\text { and concern equipment needs and sched- } \\
\text { uling. Misattribution and harsh language } \\
\text { cause conflict transformation }\end{array}$ & Very little & Yes \\
\hline $\begin{array}{l}\text { Rolloff } \\
\text { (2006) [81] }\end{array}$ & $\begin{array}{l}\text { Acquisition, } \\
\text { expression and } \\
\text { application of }\end{array}$ & $\begin{array}{l}\text { Education } \\
\text { (professional } \\
\text { training of nurses) }\end{array}$ & Focus on Nurses & Qualitative & Literature review & $\begin{array}{l}\text { A constructivist approach to the } \\
\text { baccalaureate nursing curriculum for } \\
\text { evidence based practice }\end{array}$ & Yes & $\begin{array}{l}\text { Sometimes } \\
\text { referred to }\end{array}$ \\
\hline
\end{tabular}


Table 1 Descriptive information for each study included in the scoping review (Continued)

\begin{tabular}{|c|c|c|c|c|c|c|c|c|}
\hline & $\begin{array}{l}\text { knowledge for } \\
\text { professional practice }\end{array}$ & & & & & & & \\
\hline $\begin{array}{l}\text { Smith } \\
\text { (2007) [42] }\end{array}$ & $\begin{array}{l}\text { Design of a KT } \\
\text { activity/intervention }\end{array}$ & $\begin{array}{l}\text { Primary health care } \\
\text { setting }\end{array}$ & Nurses & Intervention & $\begin{array}{l}\text { Compare } 2 \\
\text { instructional design } \\
\text { strategies in pain } \\
\text { management }\end{array}$ & $\begin{array}{l}\text { Constructivist design took more time, no } \\
\text { difference between constructivist and } \\
\text { traditional design, learner satisfaction with } \\
\text { online experience }\end{array}$ & Yes & Yes \\
\hline $\begin{array}{l}\text { Schluter } \\
\text { (2011) [60] }\end{array}$ & $\begin{array}{l}\text { Acquisition, } \\
\text { expression and } \\
\text { application of } \\
\text { knowledge for } \\
\text { professional practice }\end{array}$ & $\begin{array}{l}\text { Primary health care } \\
\text { setting }\end{array}$ & Medical and surgical nurses & Qualitative & Interviews & $\begin{array}{l}\text { Limits of scope of practice between } \\
\text { different nursing practices }\end{array}$ & Yes & Yes \\
\hline $\begin{array}{l}\text { Tilleczek } \\
\text { (2005) [43] }\end{array}$ & $\begin{array}{l}\text { Design of a } \mathrm{KT} \\
\text { activity/intervention }\end{array}$ & $\begin{array}{l}\text { Education (post- } \\
\text { graduate training of } \\
\text { health care } \\
\text { professionals) }\end{array}$ & Nurses & Intervention & $\begin{array}{l}\text { Online course and } \\
\text { survey }\end{array}$ & $\begin{array}{l}\text { Increased knowledge and skills, confidence } \\
\text { in daily practice. Learners appreciated } \\
\text { flexibility of online learning }\end{array}$ & Yes & No \\
\hline $\begin{array}{l}\text { Varpio } \\
\text { (2006) [82] }\end{array}$ & $\begin{array}{l}\text { Acquisition, } \\
\text { expression and } \\
\text { application of } \\
\text { knowledge for } \\
\text { professional practice }\end{array}$ & $\begin{array}{l}\text { Primary health care } \\
\text { setting }\end{array}$ & $\begin{array}{l}\text { Physicians and nurses, both } \\
\text { novice and experts using } \\
\text { electronic patient records }\end{array}$ & Qualitative & $\begin{array}{l}\text { Non participant } \\
\text { observation and } \\
\text { interviews }\end{array}$ & $\begin{array}{l}\text { Electronic patient records were printed } \\
\text { and the information modified, as it did not } \\
\text { facilitate professional work activities. }\end{array}$ & No & No \\
\hline $\begin{array}{l}\text { Wilson } \\
\text { (2000) [51] }\end{array}$ & $\begin{array}{l}\text { Meaning of } \\
\text { 'evidence', tension } \\
\text { between research } \\
\text { and practice }\end{array}$ & N/A & Focus on biomedicine & Qualitative & $\begin{array}{l}\text { Literature review / } \\
\text { reflection }\end{array}$ & $\begin{array}{l}\text { Biomedicine model, debate of } \\
\text { effectiveness of objectivism approach in } \\
\text { health care vs. subjectivist model, which } \\
\text { includes the new emerging theory of SC }\end{array}$ & Yes & Yes \\
\hline
\end{tabular}


Table 2 Study designs of included studies

\begin{tabular}{|c|c|c|}
\hline $\begin{array}{l}\text { Study design and } \\
\mathrm{N} \text { of studies }\end{array}$ & Method & Number of studies \\
\hline \multirow{4}{*}{$\begin{array}{l}\text { Intervention (mixed } \\
\text { experimental) }\end{array}$} & \multirow{2}{*}{$\begin{array}{l}\text { Workshop or course } \\
\text { followed by survey }\end{array}$} & 5 \\
\hline & & {$[38-41,43]$} \\
\hline & \multirow{2}{*}{$\begin{array}{l}\text { Comparison of different } \\
\text { learning programs }\end{array}$} & 1 \\
\hline & & [42] \\
\hline \multirow{4}{*}{$\begin{array}{l}\text { Mixed (qualitative } \\
\text { and quantitative) }\end{array}$} & \multirow{2}{*}{$\begin{array}{l}\text { Focus Group and } \\
\text { quantitative analysis }\end{array}$} & 1 \\
\hline & & [44] \\
\hline & \multirow{2}{*}{$\begin{array}{l}\text { Workshop and reflective } \\
\text { practice/discussion }\end{array}$} & 1 \\
\hline & & {$[45]$} \\
\hline \multirow[t]{8}{*}{ Qualitative } & \multirow[t]{2}{*}{ Action Group } & 1 \\
\hline & & {$[79]$} \\
\hline & \multirow[t]{2}{*}{ Focus group } & 1 \\
\hline & & {$[64]$} \\
\hline & \multirow[t]{2}{*}{ Interview/Questionnaire } & 8 \\
\hline & & {$[53,59-63,65,76]$} \\
\hline & \multirow[t]{2}{*}{ Observation/Interview } & 1 \\
\hline & & {$[82]$} \\
\hline \multirow[t]{5}{*}{ Other } & \multirow{2}{*}{$\begin{array}{l}\text { Editorial Opinion Or } \\
\text { Reflective paper }\end{array}$} & 5 \\
\hline & & {$[47,54,57,77,80]$} \\
\hline & \multirow[t]{3}{*}{ Literature review } & 11 \\
\hline & & {$[46,48-52,55,56,58,78,82]$} \\
\hline & & TOTAL 35 \\
\hline
\end{tabular}

[39,53-60,77,78,80-82]. Nine of the 14 papers corresponding to this theme were literature reviews, conceptual papers and reflective pieces [54-58,77,78,80,81]. Topics were varied and ranged from how factors such as loyalty to the profession has an impact on practice [53],

Table 3 Practice settings of included studies

\begin{tabular}{ll}
\hline Practice settings & Number of studies \\
\hline Primary health care setting & 10 \\
Health, health promotion, & {$[42,44,53,59-62,64,65,82]$} \\
and health services & 2 \\
organizations & {$[38,48]$} \\
Post-graduate education & 7 \\
& {$[39-41,43,45,65,81]$} \\
Mental Health services & 5 \\
& {$[50,52,58,78,80]$} \\
Mental health services, & 1 \\
social, and community & {$[76]$} \\
services & 1 \\
Home care programs & {$[79]$} \\
& 9 \\
Not applicable & {$[46,47,4449,51[55-57,77]$} \\
\hline
\end{tabular}

Table 4 Participants/professional groups of included studies

\begin{tabular}{ll}
\hline Participants/professional groups & Number of studies \\
\hline Nurses & 9 \\
& {$[42,43,47,53,55,60-62,77]$} \\
Nurses and patients & 1 \\
& {$[44]$} \\
Nurses and physicians & 2 \\
Interdisciplinary team & {$[64,82]$} \\
\end{tabular}

$[38,39,48,51,57,65,76,79]$ Physicians 2

$[46,59]$

Health services researchers $\quad 1$

\begin{tabular}{ll} 
& {$[54]$} \\
Physical therapists & 2 \\
& {$[49,56]$} \\
Psychology/psychiatry health care & 5 \\
professionals and researchers & {$[50,52,58,78,80]$} \\
Patients & 1 \\
& {$[63]$} \\
Post-graduate (health care & 4 \\
professional training) & {$[40,41,45,80]$} \\
\hline
\end{tabular}

to how tailoring information to practice needs influences learning and learner satisfaction [39].

Major findings from the review papers included the notion that the social constructivist paradigm provides a means for professionals to voice their knowledge [54], the importance of context in learning and the difficulty in transferring knowledge to different contexts in physical therapy practice [55]. Higgs [56] explored the nature of knowledge and discussed knowledge as 'underpinning clinical practice'. He suggested that knowledge is an active and dynamic phenomenon constantly undergoing changes and being tested in practice. In addition, Higgs' paper emphasized that knowledge is the basis for evidence-based practice, as clinicians draw from experiential and declarative sources of knowledge in their daily practice. Kinsella [57] discussed the philosophical underpinnings of reflective practice and how these can be used to advance our knowledge and interpretation of practice. McGuckin's [58] literature review focused on the most effective methods for teaching modern psychiatric practice knowledge, attitudes, and skills. The author found that constructivist learning is an eclectic approach with great potential as learners are actively engaged in the learning process and they bring their unique perspectives to the learning situation. Moreover, relevant and meaningful learning activities are used to promote the desired knowledge and skills. A qualitative study of 
Table $\mathbf{5}$ Aspects of theory used in studies

\begin{tabular}{|c|c|c|c|c|c|c|c|c|}
\hline $\begin{array}{l}\text { Primary } \\
\text { author }\end{array}$ & Abad-Corpa, E [44] & Caley, L [38] & Cronin, M [45] & Daley, B [39] & Fagan, D [62] & Fairweather, C [61] & Felton, B [76] & Fonville, A [53] \\
\hline $\begin{array}{l}\text { Theory } \\
\text { used }\end{array}$ & $\begin{array}{l}\text { Participatory action } \\
\text { research design from } \\
\text { a qualitative } \\
\text { methodological } \\
\text { perspective, using } \\
\text { Checkland's 'Soft } \\
\text { Systems' theoretical } \\
\text { framework }\end{array}$ & $\begin{array}{l}\text { Participatory action } \\
\text { research design }\end{array}$ & $\begin{array}{l}\text { Rootman et al., } \\
\text { Freire et al. }\end{array}$ & $\begin{array}{l}\text { Linking new to } \\
\text { past experiences, } \\
\text { probing deeply in } \\
\text { past experiences }\end{array}$ & $\begin{array}{l}\text { The research study } \\
\text { was undertaken via a } \\
\text { constructivist } \\
\text { paradigm. }\end{array}$ & $\begin{array}{l}\text { The study was guided } \\
\text { by the methodology } \\
\text { of constructivism. This } \\
\text { approach to } \\
\text { qualitative inquiry is } \\
\text { based on the } \\
\text { assumption that in } \\
\text { order to gain an } \\
\text { understanding of the } \\
\text { social world we need } \\
\text { to examine it from } \\
\text { the perspective of } \\
\text { those who arc the } \\
\text { active participants in } \\
\text { that world. }\end{array}$ & $\begin{array}{l}\text { The case study } \\
\text { described in this } \\
\text { paper used a } \\
\text { 'constructivist' } \\
\text { methodology, that is, } \\
\text { a research technique } \\
\text { that utilizes key } \\
\text { actors' and close } \\
\text { observers' } \\
\text { understandings and } \\
\text { interpretations of the } \\
\text { implementation } \\
\text { (Guba and Lincoln, } \\
\text { 1989). }\end{array}$ & $\begin{array}{l}\text { Constructivist } \\
\text { paradigm }\end{array}$ \\
\hline $\begin{array}{l}\text { Aspects } \\
\text { of } \\
\text { theory } \\
\text { used }\end{array}$ & $\begin{array}{l}\text { Social environment } \\
\text { plays a critical role in } \\
\text { the development of } \\
\text { knowledge. }\end{array}$ & $\begin{array}{l}\text { Social environment } \\
\text { plays a critical role in } \\
\text { the development of } \\
\text { knowledge. }\end{array}$ & $\begin{array}{l}\text { Social environment } \\
\text { plays a critical role in } \\
\text { the development of } \\
\text { knowledge. }\end{array}$ & $\begin{array}{l}\text { Learning is a result } \\
\text { of the individual's } \\
\text { interaction with } \\
\text { the environment }\end{array}$ & $\begin{array}{l}\text { Cognitive dissonance } \\
\text { as the stimulus for } \\
\text { learning }\end{array}$ & $\begin{array}{l}\text { Learning is a result of } \\
\text { the individual's } \\
\text { interaction with the } \\
\text { environment }\end{array}$ & $\begin{array}{l}\text { Learning is a result of } \\
\text { the individual's } \\
\text { interaction with the } \\
\text { environment }\end{array}$ & $\begin{array}{l}\text { Learning is a result of } \\
\text { the individual's } \\
\text { interaction with the } \\
\text { environment }\end{array}$ \\
\hline $\begin{array}{l}\text { KTA } \\
\text { phase }\end{array}$ & $\begin{array}{l}\text { Step 4: Select, tailor } \\
\text { and implement } \\
\text { intervention }\end{array}$ & $\begin{array}{l}\text { Step 4: Select, tailor } \\
\text { and implement } \\
\text { intervention }\end{array}$ & $\begin{array}{l}\text { Step 4: Select, tailor } \\
\text { and implement } \\
\text { intervention }\end{array}$ & $\begin{array}{l}\text { Step 3: Assessing } \\
\text { barriers and } \\
\text { facilitators }\end{array}$ & $\begin{array}{l}\text { Step 3: Assessing } \\
\text { barriers and facilitators }\end{array}$ & $\begin{array}{l}\text { Step 3: Assessing } \\
\text { barriers and facilitators }\end{array}$ & $\begin{array}{l}\text { Step 2: Adapting } \\
\text { knowledge to local } \\
\text { context }\end{array}$ & $\begin{array}{l}\text { Step 1: Identify } \\
\text { problem }\end{array}$ \\
\hline $\begin{array}{l}\text { Primary } \\
\text { author }\end{array}$ & Lipman, T [59] & Smith, C [42] & Tilleczek, K [43] & Varpio, L [82] & $\begin{array}{l}\text { Greenslade, } \\
2010 \text { [63] }\end{array}$ & $\begin{array}{l}\text { McWilliam, } \\
2009 \text { [79] }\end{array}$ & Rogers, 2011 [64] & Schluter, 2011 [60] \\
\hline $\begin{array}{l}\text { Theory } \\
\text { used }\end{array}$ & $\begin{array}{l}\text { Constructivism } \\
\text { approach }\end{array}$ & $\begin{array}{l}\text { Learning } \\
\text { Constructivism Theory }\end{array}$ & $\begin{array}{l}\text { Contructivism } \\
\text { approach - general }\end{array}$ & $\begin{array}{l}\text { Constructivist } \\
\text { grounded theory }\end{array}$ & $\begin{array}{l}\text { Constructivist } \\
\text { approach with in- } \\
\text { depth interviews and } \\
\text { comparative analysis } \\
\text { to develop and sys- } \\
\text { temically organize } \\
\text { data into four major } \\
\text { interrelated themes } \\
\text { and a connecting es- } \\
\text { sential thread. }\end{array}$ & $\begin{array}{l}\text { Constructivism } \\
\text { approach }\end{array}$ & $\begin{array}{l}\text { A constructivist } \\
\text { grounded theory } \\
\text { approach was } \\
\text { adopted for this study } \\
\text { on the basis that it } \\
\text { would allow for the } \\
\text { use of sensitising } \\
\text { concepts or guiding } \\
\text { interests derived from } \\
\text { the conflict literature, } \\
\text { as well as an } \\
\text { investigation of the } \\
\text { features of conflict } \\
\text { unique to the OR } \\
\text { team. }\end{array}$ & $\begin{array}{l}\text { Situated within a } \\
\text { constructivist } \\
\text { methodology that } \\
\text { considered individual } \\
\text { experiences, abilities, } \\
\text { and knowledge in } \\
\text { the construction of } \\
\text { scope of practice }\end{array}$ \\
\hline $\begin{array}{l}\text { Aspects } \\
\text { of } \\
\text { theory } \\
\text { used }\end{array}$ & $\begin{array}{l}\text { Social environment } \\
\text { plays a critical role in } \\
\text { the development of } \\
\text { knowledge. }\end{array}$ & $\begin{array}{l}\text { Learning is a result of } \\
\text { the individual's } \\
\text { interaction with the } \\
\text { environment }\end{array}$ & $\begin{array}{l}\text { Learning is a result of } \\
\text { the individual's } \\
\text { interaction with the } \\
\text { environment }\end{array}$ & $\begin{array}{l}\text { Learning is a result } \\
\text { of the individual's } \\
\text { interaction with } \\
\text { the environment }\end{array}$ & $\begin{array}{l}\text { Learning is a result of } \\
\text { the individual's } \\
\text { interaction with the } \\
\text { environment }\end{array}$ & $\begin{array}{l}\text { Learning is a result of } \\
\text { the individual's } \\
\text { interaction with the } \\
\text { environment }\end{array}$ & $\begin{array}{l}\text { Social environment } \\
\text { plays a critical role in } \\
\text { the development of } \\
\text { knowledge. }\end{array}$ & $\begin{array}{l}\text { Learning is a result of } \\
\text { the individual's } \\
\text { interaction with the } \\
\text { environment }\end{array}$ \\
\hline $\begin{array}{l}\text { KTA } \\
\text { phase }\end{array}$ & $\begin{array}{l}\text { Step 1: Identify } \\
\text { problem }\end{array}$ & & & & $\begin{array}{l}\text { Step 3: Assessing } \\
\text { barriers and facilitators }\end{array}$ & $\begin{array}{l}\text { Step 1: Identify } \\
\text { problem }\end{array}$ & & \\
\hline
\end{tabular}

phase problem 
Table 5 Aspects of theory used in studies (Continued)

\begin{tabular}{|c|c|c|c|c|c|c|c|c|}
\hline & & $\begin{array}{l}\text { Step 4: Select, tailor } \\
\text { and implement } \\
\text { intervention }\end{array}$ & $\begin{array}{l}\text { Step 4: Select, tailor } \\
\text { and implement } \\
\text { intervention }\end{array}$ & $\begin{array}{l}\text { Step 3: Assessing } \\
\text { barriers and } \\
\text { facilitators }\end{array}$ & & & $\begin{array}{l}\text { Step 2: Adapting } \\
\text { knowledge to local } \\
\text { context }\end{array}$ & $\begin{array}{l}\text { Step 3: Assessing } \\
\text { barriers and } \\
\text { facilitators }\end{array}$ \\
\hline $\begin{array}{l}\text { Primary } \\
\text { author }\end{array}$ & Adler, R [46] & Carr, S [54] & Field, D [55] & Greenhalgh,T [65] & Higgs, J [56] & Holtslander, L [77] & Hoshmand, L [51] & Hunter, J [40] \\
\hline $\begin{array}{l}\text { Theory } \\
\text { used }\end{array}$ & $\begin{array}{l}\text { Theories and } \\
\text { definitions of } \\
\text { evidence based on } \\
\text { Descartes, Locke's } \\
\text { theory of 'tabula rasa; } \\
\text { Hume, von Uexkull - } \\
\text { Merk-Mal theory; } \\
\text { Ginzburg; Glaserfeld's } \\
\text { understanding of } \\
\text { constructivism in } \\
\text { knowledge; clinical } \\
\text { examples to illustrate } \\
\text { models of organisms } \\
\text { (Richter; Wolf and } \\
\text { Wolff) }\end{array}$ & $\begin{array}{l}\text { Highlights the } \\
\text { potential value and } \\
\text { contribution of } \\
\text { hermeneutic } \\
\text { phenomenology and } \\
\text { constructivist } \\
\text { approaches to } \\
\text { exploring and } \\
\text { knowing nursing as a } \\
\text { means to addressing } \\
\text { some of the practice } \\
\text { learning challenges }\end{array}$ & $\begin{array}{l}\text { learning is a mental } \\
\text { process, in terms of } \\
\text { the con-structivist } \\
\text { view of learning or } \\
\text { whether it owes more } \\
\text { to enculturation into } \\
\text { social processes as } \\
\text { with the situated } \\
\text { learning and legitim- } \\
\text { ate peripheral partici- } \\
\text { pation approaches to } \\
\text { learning }\end{array}$ & $\begin{array}{l}\text { Although we } \\
\text { believe the } \\
\text { constructivist } \\
\text { approach has } \\
\text { general validity, it } \\
\text { is particularly } \\
\text { appropriate for the } \\
\text { promotion of the } \\
\text { knowledge and } \\
\text { skills for } \\
\text { knowledge } \\
\text { translation. }\end{array}$ & $\begin{array}{l}\text { In this paper, the } \\
\text { critical question of } \\
\text { knowledge as the } \\
\text { underpinning of } \\
\text { clinical practice is } \\
\text { examined. The nature } \\
\text { of knowledge is } \\
\text { explored in this paper, } \\
\text { with support being } \\
\text { given to the } \\
\text { constructivisit } \\
\text { perspective }\end{array}$ & Constructivism & $\begin{array}{l}\text { Constructivism } \\
\text { (Berger and } \\
\text { Luckmann, 1966; } \\
\text { Bruffee, 1986; K. J. } \\
\text { Gergen, 1985) calls for } \\
\text { multiple paradigms of } \\
\text { knowledge. The } \\
\text { potential of multiple } \\
\text { rationalities and } \\
\text { methods of } \\
\text { construction is } \\
\text { recognized by the } \\
\text { cognitive } \\
\text { interpretation of } \\
\text { science }\end{array}$ & $\begin{array}{l}\text { Constructivist } \\
\text { learning theory was } \\
\text { an appropriate } \\
\text { conceptual } \\
\text { framework for the } \\
\text { course as it } \\
\text { acknowledges } \\
\text { multiple, socially } \\
\text { constructed truths, } \\
\text { perspectives, and } \\
\text { realities versus a } \\
\text { single reality }\end{array}$ \\
\hline $\begin{array}{l}\text { Aspects } \\
\text { of } \\
\text { theory } \\
\text { used }\end{array}$ & All 3 aspects & $\begin{array}{l}\text { Learning is a result of } \\
\text { the individual's } \\
\text { interaction with the } \\
\text { environment }\end{array}$ & $\begin{array}{l}\text { Social environment } \\
\text { plays a critical role in } \\
\text { the development of } \\
\text { knowledge. }\end{array}$ & $\begin{array}{l}\text { Learning is a result } \\
\text { of the individual's } \\
\text { interaction with } \\
\text { the environment }\end{array}$ & All 3 aspect & All 3 aspects & All 3 aspects & All 3 aspects \\
\hline $\begin{array}{l}\text { KTA } \\
\text { phase }\end{array}$ & $\begin{array}{l}\text { Knowledge creation: } \\
\text { knowledge synthesis }\end{array}$ & $\begin{array}{l}\text { Knowledge creation: } \\
\text { knowledge inquiry }\end{array}$ & $\begin{array}{l}\text { Knowledge creation: } \\
\text { knowledge synthesis }\end{array}$ & $\begin{array}{l}\text { Step 4: Select, } \\
\text { tailor and } \\
\text { implement } \\
\text { intervention }\end{array}$ & $\begin{array}{l}\text { Knowledge creation: } \\
\text { knowledge inquiry }\end{array}$ & $\begin{array}{l}\text { Knowledge creation: } \\
\text { knowledge synthesis }\end{array}$ & $\begin{array}{l}\text { Step 1: Identify } \\
\text { problem }\end{array}$ & $\begin{array}{l}\text { Step 4: Select, tailor } \\
\text { and implement } \\
\text { intervention }\end{array}$ \\
\hline $\begin{array}{l}\text { Primary } \\
\text { author }\end{array}$ & Kinsella, E [57] & Labonte, R [48] & Lyddon, W [78] & McGuckin, C [58] & Miller, K [50] & Neimeyer, R [80] & Plack, M [49] & Rogal, S [41] \\
\hline $\begin{array}{l}\text { Theory } \\
\text { used }\end{array}$ & $\begin{array}{l}\text { The constructivist } \\
\text { perspective is } \\
\text { founded on the idea } \\
\text { that humans actively } \\
\text { construct their } \\
\text { personal realities and } \\
\text { create their own } \\
\text { representational } \\
\text { models of the world' }\end{array}$ & $\begin{array}{l}\text { This article argues } \\
\text { further that a } \\
\text { 'constructivist' } \\
\text { research paradigm } \\
\text { not only has the } \\
\text { potential to resolve } \\
\text { some of the tensions } \\
\text { between research and } \\
\text { practice in health } \\
\text { promotion but also is } \\
\text { inclusive of } \\
\text { knowledge generated } \\
\text { by the conventional } \\
\text { paradigm. }\end{array}$ & $\begin{array}{l}\text { Constructivism } \\
\text { approach in general }\end{array}$ & $\begin{array}{l}\text { Constructivist } \\
\text { learning is based } \\
\text { on an eclectic mix } \\
\text { of ideas derived } \\
\text { primarily from } \\
\text { cognitive } \\
\text { neuroscience } \\
\text { including } \\
\text { information } \\
\text { processing theory. }\end{array}$ & $\begin{array}{l}\text { Constructivism } \\
\text { emphasizes the } \\
\text { socially constructed } \\
\text { nature of reality; it } \\
\text { shifts attention away } \\
\text { from the search for } \\
\text { universal truths and } \\
\text { toward an exploration } \\
\text { of what is considered } \\
\text { real within particular } \\
\text { social contexts. }\end{array}$ & $\begin{array}{l}\text { In sharp contrast to } \\
\text { this worldview, social } \\
\text { constructivism } \\
\text { endorses a form of } \\
\text { postmodernism } \\
\text { (Anderson, 1990) that } \\
\text { turns nearly every } \\
\text { aspect of this modern } \\
\text { psychological } \\
\text { program on its head. } \\
\text { Gone is the faith in an } \\
\text { objectively knowable } \\
\text { universe, and with it } \\
\text { the hope that } \\
\text { elimination of human } \\
\text { bias, adherence to } \\
\text { canons of }\end{array}$ & $\begin{array}{l}\text { The constructivist } \\
\text { emphasizes the } \\
\text { personal meaning } \\
\text { made by the inquirer } \\
\text { and the inquired. }\end{array}$ & $\begin{array}{l}\text { Constructivism } \\
\text { relates to the } \\
\text { philosophy that the } \\
\text { meaning of new } \\
\text { learning is } \\
\text { constructed upon } \\
\text { current knowledge }\end{array}$ \\
\hline
\end{tabular}




\begin{tabular}{|c|c|c|c|c|c|c|c|c|}
\hline \multirow[b]{2}{*}{$\begin{array}{l}\text { Aspects } \\
\text { of } \\
\text { theory } \\
\text { used }\end{array}$} & \multirow[b]{2}{*}{$\begin{array}{l}\text { Cognitive dissonance } \\
\text { as the stimulus for } \\
\text { learning }\end{array}$} & \multirow[b]{2}{*}{$\begin{array}{l}\text { Cognitive dissonance } \\
\text { as the stimulus for } \\
\text { learning }\end{array}$} & \multirow[b]{2}{*}{$\begin{array}{l}\text { Cognitive dissonance } \\
\text { as the stimulus for } \\
\text { learning }\end{array}$} & \multirow[b]{2}{*}{$\begin{array}{l}\text { Social environment } \\
\text { plays a critical role } \\
\text { in the } \\
\text { development of } \\
\text { knowledge }\end{array}$} & \multirow[b]{2}{*}{$\begin{array}{l}\text { Learning is a result of } \\
\text { the individual's } \\
\text { interaction with the } \\
\text { environment }\end{array}$} & \\
\hline & & & & & & All 3 & All 3 & $\begin{array}{l}\text { Learning is a result of } \\
\text { the individual's } \\
\text { interaction with the } \\
\text { environment }\end{array}$ \\
\hline $\begin{array}{l}\text { KTA } \\
\text { phase }\end{array}$ & $\begin{array}{l}\text { Knowledge creation: } \\
\text { knowledge synthesis }\end{array}$ & $\begin{array}{l}\text { Knowledge creation: } \\
\text { knowledge synthesis }\end{array}$ & $\begin{array}{l}\text { Knowledge creation: } \\
\text { knowledge tools/ } \\
\text { products }\end{array}$ & $\begin{array}{l}\text { Step 3: Assessing } \\
\text { barriers and } \\
\text { facilitators }\end{array}$ & $\begin{array}{l}\text { Knowledge creation: } \\
\text { knowledge inquiry }\end{array}$ & $\begin{array}{l}\text { Knowledge creation: } \\
\text { knowledge inquiry }\end{array}$ & $\begin{array}{l}\text { Knowledge creation: } \\
\text { knowledge synthesis }\end{array}$ & $\begin{array}{l}\text { Step 4: Select, tailor } \\
\text { and implement } \\
\text { intervention }\end{array}$ \\
\hline $\begin{array}{l}\text { Primary } \\
\text { author }\end{array}$ & Rolloff, M [81] & Wilson, H [51] & Appleton, J. [47] & & & & & \\
\hline $\begin{array}{l}\text { Theory } \\
\text { used }\end{array}$ & $\begin{array}{l}\text { Constructivism } \\
\text { assumes that learners } \\
\text { construct knowledge } \\
\text { as part of a process of } \\
\text { making sense of their } \\
\text { experiences: 'Learners, } \\
\text { therefore, are not } \\
\text { empty vessels waiting } \\
\text { to be filled, but rather } \\
\text { active organisms } \\
\text { seeking meaning' } \\
\text { (Driscoll, 2005, p. 387). }\end{array}$ & $\begin{array}{l}\text { The underlying } \\
\text { science here is } \\
\text { located in a } \\
\text { constructivist } \\
\text { philosophy while } \\
\text { other descriptive } \\
\text { terms would be } \\
\text { phenomenological, } \\
\text { interpretivist or } \\
\text { subjectivist }\end{array}$ & $\begin{array}{l}\text { Philosophical } \\
\text { underpinnings of } \\
\text { constructivism, post- } \\
\text { positivism, critical real- } \\
\text { ism (in terms of realis- } \\
\text { tic evaluation) and } \\
\text { participatory inquiry }\end{array}$ & & & & & \\
\hline $\begin{array}{l}\text { Aspects } \\
\text { of } \\
\text { theory } \\
\text { used }\end{array}$ & $\begin{array}{l}\text { Learning is a result of } \\
\text { the individual's } \\
\text { interaction with the } \\
\text { environment }\end{array}$ & $\begin{array}{l}\text { Social environment } \\
\text { plays a critical role in } \\
\text { the development of } \\
\text { knowledge. }\end{array}$ & All 3 & & & & & \\
\hline $\begin{array}{l}\text { KTA } \\
\text { phase }\end{array}$ & $\begin{array}{l}\text { Knowledge creation: } \\
\text { knowledge synthesis }\end{array}$ & $\begin{array}{l}\text { Step 3: Assessing } \\
\text { barriers and } \\
\text { facilitators }\end{array}$ & $\begin{array}{l}\text { Knowledge creation: } \\
\text { knowledge inquiry }\end{array}$ & & & & & \\
\hline
\end{tabular}


Table 6 Main themes and major concepts emerging from the application of social constructivist theory to knowledge translation interventions

\begin{tabular}{|c|c|c|c|c|}
\hline THEME 1 & THEME 2 & THEME 3 & THEME 4 & THEME 5 \\
\hline Meaning of 'evidence' & $\begin{array}{l}\text { Understanding acquisition, } \\
\text { expression and application of } \\
\text { knowledge in and for } \\
\text { professional practice }\end{array}$ & $\begin{array}{l}\text { Promoting professional } \\
\text { expertise as a component } \\
\text { of evidence-based practice }\end{array}$ & $\begin{array}{l}\text { Understanding } \\
\text { clients and their } \\
\text { experiences }\end{array}$ & $\begin{array}{l}\text { Designing interventions to a) } \\
\text { increase knowledge and skill } \\
\text { acquisition; b) change } \\
\text { behaviour }\end{array}$ \\
\hline $\begin{array}{l}\text { Post-modernist views on } \\
\text { knowledge and knowledge } \\
\text { acquisition }\end{array}$ & Practice based on experience & $\begin{array}{l}\text { Knowledge of ethics and } \\
\text { professional practice }\end{array}$ & $\begin{array}{l}\text { Outcomes } \\
\text { regarding patient } \\
\text { care }\end{array}$ & For generating EBP knowledge \\
\hline $\begin{array}{l}\text { Original/authentic problems } \\
\text { to be addressed }\end{array}$ & $\begin{array}{l}\text { Role of personal and } \\
\text { professional values vs. formal } \\
\text { knowledge }\end{array}$ & Novice vs. expert & $\begin{array}{l}\text { Understanding of } \\
\text { clients' realities }\end{array}$ & For sharing knowledge \\
\hline \multirow[t]{13}{*}{ Meaning of evidence } & Experiential learning & Progress and role & & $\begin{array}{l}\text { For impacting on knowledge, } \\
\text { attitudes and intentions to apply } \\
\text { o evidence in practice }\end{array}$ \\
\hline & Meaning of experiences & $\begin{array}{l}\text { SC in how learning and } \\
\text { expertise develop }\end{array}$ & & $\begin{array}{l}\text { For promoting reflective } \\
\text { practice }\end{array}$ \\
\hline & Patient welfare as a motivator & $\begin{array}{l}\text { Differences between specialist } \\
\text { and generalist in skills and } \\
\text { knowledge }\end{array}$ & & $\begin{array}{l}\text { For problem solving, critical } \\
\text { thinking and reflection }\end{array}$ \\
\hline & Perceived support & Combining experiences & & For changing attitudes \\
\hline & Loyalty to profession & Working together & & For creating meaning \\
\hline & $\begin{array}{l}\text { Learner satisfaction and } \\
\text { involvement }\end{array}$ & & & $\begin{array}{l}\text { For sharing knowledge } \\
\text { (Theoretical and practical) }\end{array}$ \\
\hline & Meaning of competency & & & $\begin{array}{l}\text { For knowledge that is } \\
\text { actionable }\end{array}$ \\
\hline & Role of previous experience & & & For practice based evaluations \\
\hline & Direct, reflective learning & & & \\
\hline & Role of context & & & \\
\hline & Feedback from colleagues & & & \\
\hline & Learners guide learning process & & & \\
\hline & Threat of evaluation & & & \\
\hline
\end{tabular}

physician researchers found that implementing research evidence is more complex than suggested in current models of evidence-based medicine and that clinical decision-making is strongly influenced by factors other than just research evidence [59]. Schluter [60] used a critical incident technique grounded in a constructivist methodology to understand how nurses conceive their scope of practice. Findings suggested that different nursing areas of expertise have diverse scopes of practice, that require varied methods for applying knowledge, with the optimal method relying on nurses' grade and skill mix.

Theme three: promoting professional expertise as a component of evidence-based practice ( $n=1$ paper) Fairweather [61] used focus groups with primary healthcare nurse specialists in order to identify the characteristics and attributes of competency that specialist nurses ascribe to their practice; describe how specialist nurses delineate specialist boundaries from generalist practice; and generate evidence based knowledge for the development of regulatory procedures for nurses. Results indicated that knowledge is a synthesis of propositional and practice knowledge and that expertise was gained through exposure and reflection. Assumptions from social constructivist theory were used to help novices move towards expertise in practice. Differences in knowledge acquisition and application were believed to be associated with level of experience and expertise.

Theme four: understanding clients and their experiences/ realities ( $n=2$ papers)

This theme focused on the use of constructivist approaches for examining the 'centrality' of the patient. The two studies in this theme found that health care professionals keep the patient at the 'center' of their clinical decisions and treatment interventions when acquiring and applying knowledge. Fagan [62] used a constructivist inquiry approach to examine accident and emergency nurses' perceptions of their roles in identifying child abuse in primary healthcare. 
The ability of nurses to identify children in potentially abusive situations required nurses to evaluate the child's safety as accurately as possible, to ensure that he/she received the appropriate treatment and attention. The author concluded that although all nurses in the study had sufficient knowledge to identify child abuse, this knowledge base increased with experience. The author also suggested that additional training and education is needed for multidisciplinary decision making about the role of nurses in this context. A qualitative study by Greenslade and Mandville-Ansey [63] used in-depth interviews within a constructivist approach to understand the experiences of women having same-day breast cancer surgery. Women's subjective experiences were used to make client-centered recommendations to assist healthcare professionals in effecting change to enhance quality of care.

Theme five: designing interventions aimed at knowledge and skill acquisition and changing behavior ( $n=9$ papers) Nine studies examined the effects of various interventions aimed at increasing knowledge and skills in order to improve practice. One study used a mixed methods design [44], one used a qualitative design [64] and the remaining seven used surveys $[38,42,43,45]$ and workshop evaluations $[40,41,65]$. The intervention studies assessed workshops that were related to specific clinical training skills such as cultural competence [40] and alcohol dependence screening [38], while some focused on social constructivism approaches as strategies for experiential learning and reflective practice [45]. In this theme, social constructivist theory was used to inform the design of $\mathrm{KT}$ interventions intended to promote core skills, knowledge, and competencies needed for evidence based practice, and support behavior change (increased use of best practices).

\section{Discussion}

The purpose of this scoping review was to examine the applications of social constructivist theory in knowledge translation for best practice in the health professions. Consistent with the findings by Colquhoun et al. [19] and Davies et al. [20], the use of social constructivist theory in the KT literature is limited and haphazard. Most papers describing results of original research neglected to justify why the use of theory was central to the research question, and most papers did not make explicit the relevance and potential applications of social constructivist theory in KT. While we acknowledge that lack of justification for theory use represents a major limitation of the papers, most $(n=28)$ were published after Colquhoun et al.'s [19] and Davies et al.'s [20] review papers. Likewise, 23 papers were published before the germinal articles by Eccles et al. [14] and the ICEBerg group [15]. Indeed these KT scholars have advocated for explicit statements regarding the use of theory in KT research $[14,15,17,19,20,66-68]$. We suggest that without at least a definition of the theory and at most, a discussion of theoretical assumptions and underpinnings, the potential for theories to guide and inform the field of KT is limited at best.

There was important variability in the study designs, areas of practice, targeted health professions, and methodological approaches used across the 35 papers. The papers ranged from reflective discussions to qualitative studies, and only six papers used an experimental design to assess the impact of various KT interventions grounded in social constructivist approaches. Such variability most likely reflects the early stages of development in the use of social constructivism as a potentially valuable theory in the field of $\mathrm{KT}$.

In terms of practice settings, we were interested in the clinical areas where the theory would be most frequently applied. Twenty-six papers reported results from five different types of clinical settings and practice areas (ten papers were from the primary healthcare setting, seven from postgraduate education environments, six from mental health service settings, one from home care and two from health services organizations). It is not clear whether this represents a heterogeneous list of settings and as such, we cannot conclude on the nature of the clinical settings most often targeted by efforts to highlight the link between social constructivism and KT.

Among the health disciplines represented in the reviewed papers, nursing accounted for one-third. This finding is consistent with the current state of $\mathrm{KT}$ research in this profession. Indeed, nursing scholars have produced much of the seminal KT literature, including key papers in KT theory $[20,69]$. While eight of the 35 papers addressed KT within interdisciplinary teams, few studies mentioned which other professions were involved or their roles in the $\mathrm{KT}$ interventions. Overall, there was representation from seven disciplines reflecting the broad spectrum of professions interested in $\mathrm{KT}$ and best practice.

Although we were able to identify which components of the KTA framework [32] were targets of the study interventions and/or discussions (Table 6), these were not addressed explicitly in any of the papers. An assumption of the KTA framework believed to be important for both researchers and practitioners is that it considers various sources of information as 'knowledge' and/or 'evidence'. These sources include knowledge from research findings as well as other forms of knowing such as experiential knowledge [32], which refers to learning from experience through reflection and is considered essential for integrating and making sense of the knowledge that emerges from scientific research [70].

According to Graham and Tetroe [34], the KTA falls within the social constructivist paradigm as it 'privileges 
social interaction and adaptation of research evidence that takes local context and culture into account...and offers a holistic view of the KT phenomenon by integrating the concepts of knowledge creation and action'. The framework underscores the fluid boundaries between knowledge creation and application as it highlights the need to create knowledge that emerges from knowledge users' questions (KTA step one), when it emphasizes the need to adapt the knowledge to the local context (KTA step two), and when it suggests that we select, tailor, and implement interventions that will facilitate the uptake of new knowledge for practitioners (KTA step four). Indeed, the authors of the KTA framework advocate for a participatory model whereby end users (e.g., clinicians, multidisciplinary teams, patients, and decision makers) are involved in developing research questions and carrying out research activities [32]. Collaborative interactions at every step of the KT process are believed to facilitate optimal use of research evidence and other forms of knowledge in clinical practice [71-73]. The KTA framework has the potential to help researchers understand the mechanisms that promote a collaborative approach to identifying knowledge gaps and the likelihood of successful interventions aimed at changing practice.

The final discussion points relate to the main contributions of social constructivism in the field of $\mathrm{KT}$ in the health professions. The focus on the meaning of evidence across health professions continues to be a dominant issue in the literature. In fact, this review has highlighted that post-modernist views of knowledge, knowledge acquisition, and knowledge construction do support the legitimacy of the various sources of evidence and their use in practice. Constructed knowledge or knowledge that emerges from a collaborative constructive process among various stakeholders in the clinical setting, can and should be considered as valid sources of evidence in conjunction with research generated evidence.

A major finding from the review involves the acquisition, expression, and application of knowledge in practice, with an emphasis on how social constructivist perspectives support clinicians in expressing this knowledge in their professional interactions. The important role of 'context' in promoting and supporting best practices, which has been emphasized by several KT scholars $[32,70,74,75]$, was also highlighted in this review. There is a clear need for future targeted research, as researchers and practitioners grapple with identifying and addressing the complex interaction of individual and contextual variables that play a key role in clinical practice. In their 1995 paper, Higgs and Titchen suggested that knowledge is active and dynamic, constantly undergoing changes and being tested in practice [56]. The notion that knowledge is dynamic in nature is congruent with the major tenets of social constructivism and should be reflected in the evaluation process of knowledge translation interventions [24,27,28]. We suggest that this idea must be assigned its rightful place in the KT discourse [31] as the dynamic nature of knowledge creation and exchange is also consistent with the core principles of the KTA framework.

Least surprising and perhaps most important, are the findings from the final theme: the use of social constructivist assumptions for designing and implementing interventions aimed at knowledge and skill acquisition and behavior change. As is the case with many other learning theories, designing environments and interventions that will foster optimal learning and behavior change represents educational best practice $[16,17,66]$. Indeed, relying on constructivist assumptions to support the design of KT interventions designed to foster changes in knowledge, skills, attitudes, and behavior is a practice that has been strongly advocated $[14,15,17,66]$.

\section{Limitations}

Scoping reviews offer a unique opportunity to retrieve and scan a broad range of literature to answer a research question. A potential limitation is that by searching the titles, abstracts, and subject headings only, we may have missed relevant papers. We could have also used citation chaining in Web of Science or Scopus to find other articles that cited the key articles we had already found.

We recommend that future research in this area clearly cite the theories used to design KT interventions to ensure that they are identified in reviews similar to this one, as well as in systematic reviews of theory.

The articles included in this review were not appraised for their scientific rigor, as scoping reviews do not typically include critical appraisals of the evidence. In deciding to summarize and report the overall findings without the scrutiny of a formal appraisal process, we recognize that our results speak to the extent of the research activity, major conclusions and research gaps, rather than provide the reader with support for the effectiveness of interventions or for evidence-informed recommendations that were grounded in the social constructivist paradigm. However, this represents the evolution of research that explicitly incorporates social constructivist theory in the development and application of KT. As a larger number of studies rigorously test $\mathrm{KT}$ interventions guided by theory, systematic review and quality appraisal will be necessary.

\section{Conclusion}

This review is the first to examine the use of social constructivism in KT studies. Results from this review contribute to discussions that are currently taking place on the use and usefulness of theory in KT. There are 
divided opinions about the value of theory in the field and about whether it is possible to have overarching theories that can be used to further solidify the science of $\mathrm{KT}$, improve practice, and ultimately improve patient care. Despite the debates over this issue, we argue that moving forward without considering the use of theory in $\mathrm{KT}$ is not sound scientific practice. Without theory, it will be difficult to understand the underlying mechanisms behind interventions, understand the impact the various interventions have on behaviour change and to compare across studies.

Our review indicates that social constructivism has not been widely explored in the field of KT. As a sociological theory of knowledge, it has the potential to illuminate how individuals use new information and knowledge to make sense of existing practices and how the meaning of the new knowledge may change as a result of an individual's existing knowledge base and the relevance of the new knowledge to existing practices.

We argued that the links between social constructivism and KT have not been fully explored but that the KTA framework has constructivist underpinnings that help the discussion on how the theory can be used in the broader KT enterprise moving forward. Indeed the KTA framework advocates for interventions that consider learning to be the result of human interactions that take place within a socially mediated context.

There will undoubtedly be many more discussions and debates about using theories to advance the science of $\mathrm{KT}$ and perhaps even more conversations about which theories are most appropriate in a given situation or context. This will become increasingly important as our knowledge base about the likelihood of successful interventions across contexts and health disciplines continues to grow. We suggest that social constructivist theories hold much promise for informing the design of $\mathrm{KT}$ interventions. In this context, social constructivist interventions will take into account that practicing clinicians are part of complex social systems, that they may privilege active collaborative strategies for knowledge acquisition and that the potential for learning is greater when the new knowledge conflicts in some way with existing practices (cognitive dissonance). Moreover, KT interventions grounded in social constructivist theories will value clinicians' prior knowledge and experiences as essential components of knowledge creation and application. These critical sources of 'evidence' will be in constant interaction with new knowledge and scientific evidence to help support clinicians in developing new understandings of clinical phenomena.

Finally, we propose that further research is needed to test the use of social constructivist assumptions in the design and implementation of KT interventions. The contribution of the theory lies in its potential to unveil the individual processes that are involved in the 'construction' and application of knowledge in clinical practice.

\section{Additional file}

Additional file 1: Full electronic search strategy for Ovid Medline (1948 - May 16, 2011).

\section{Competing interests}

The authors declare that they have no competing interests.

\section{Authors contributions}

All authors made substantial contributions to conception and design, or acquisition of data, or analysis and interpretation of data. All authors were also involved in drafting and revising the manuscript for important intellectual content. All authors have given final approval of the version to be published.

\section{Funding}

This scoping review was supported by the Edith Strauss Rehabilitation Research Projects, a knowledge translation initiative funded by the Richard and Edith Strauss Foundation of Canada and supported by the School of Physical and Occupational Therapy, McGill University. The following present committee members contributed to the project (some of which are authors of this manuscript): Sara Ahmed PhD, Genevieve Côté-Leblanc OT, Erika Hasler OT, Nicol Korner-Bitensky PhD, Annette Majnemer PhD, Nancy Mayo PhD, Ana Maria Rodriguez PhD, Anita Menon PhD, Laurie Snider PhD, Aliki Thomas PhD, and Diana Valentini PT. We would like to acknowledge Lucy Li for her assistance in formatting this manuscript and Drs. lan Graham and Andre Bussieres for their valuable feedback on earlier versions of the manuscript.

\section{Author details}

${ }^{1}$ School of Physical and Occupational Therapy, McGill University, Montreal, Quebec, Canada. ${ }^{2}$ Centre for Interdisciplinary Rehabilitation Research of Greater Montreal, Montreal, Quebec, Canada. ${ }^{3}$ Centre for Medical Education, Faculty of Medicine, McGill University, Montreal, Quebec, Canada. ${ }^{4}$ Life Sciences Library, Faculty of Medicine, McGill University, Montréal, Quebec, Canada.

Received: 14 July 2013 Accepted: 1 May 2014

Published: 6 May 2014

\section{References}

1. Canadian Physiotherapy Association: 2012. http://www.physiotherapy.ca/PracticeResources.

2. Canadian Association for Occupational Therapists: Continuing professional education. Continuing professional education; 2012. http://www.caot.ca/ CAOT_career_listings.asp?pageid=14.

3. Sackett DL, Rosenberg WM, Gray JA, Haynes BR, Richardson SW: Evidence based medicine: what it is and what it isn't. BMJ 1996, 312:71-72.

4. Duncan PW, Horner RD, Reker DM, Samasa GP, Hoenig H, Hamilton B, LaClair B, Dudley TK: Adherence to post-acute rehabilitation guidelines is assosciated with functional recovery in stroke. Stroke 2002, 33:167-178.

5. Davis D, Evans M, Jadad A, Perrier L, Rath D, Ryan D, Sibbald G, Straus S, Rappolt S, Wowk M, Zwarenstein M: The case for knowledge translation: shortening the journey from evidence to effect. BMJ 2003, 327:33-35.

6. McGlynn EA, Asch SM, Adams J, Keesey J, Hicks J, DeCristofaro A: The quality of health care delivered to adults in the United States. N Engl J Med 2003, 348:2635-2645.

7. Grol R: Successes and failures in the implementation of evidence-based guidelines for clinical practice. Med Care 2001, 39:1146-1154.

8. Korner-Bitensky N, Wood-Dauphinee S, Teasell R, Hanley J, Desrosiers J, Malouin F, Thomas A, Harrison M, Kaizer F, Kehayia E, Levin M, Jutai J, Menon-Nair A, Fung J, Richards C, Dumoulin C, Rochette A, Kloda L, Martino R, Mayo N, Eng J, Duncan $P$, Page S: Best versus Actual Practices in Stroke Rehabilitation: Results of the Canadian National Survey. 6th World Stroke Congress. Stroke 2006, 37:631. 
9. Dumoulin C, Korner-Bitensky N, Tannenbaum C: The cross Canada study of stroke rehabilitation: finding on the identification, assessment and management of urinary incontinence after stroke. Stroke 2007, 38:2745-2751.

10. Korner-Bitensky N, Desrosiers J, Rochette A: A national survey of occupational therapists' practices related to participation post-stroke. J Rehab Med 2008, 37:513-519.

11. Menon-Nair A, Korner-Bitensky N, Ogourtsova T: Occupational therapists' identification, assessment and treatment of unilateral spatial neglect during stroke rehabilitation in Canada. Stroke 2007, 38:2556-2562.

12. Canadian Institutes of Health Research: Knowledge Translation. Knowledge Translation; 2013. http://www.cihr-irsc.gc.ca/e/39033.html.

13. Philippa D, Walker A, Grimshaw J: A systematic review of the use of theory in the design of guideline dissemination and implementation strategies and interpretation of the results of rigorous evaluations. Implementation Sci 2010, 5:14

14. Eccles M, Grimshaw J, Walker A, Johnston M, Pitts N: Changing the behavior of healthcare professionals: the use of theory in promoting the uptake of research findings. J Clin Epidemol 2005, 58:107-112.

15. ICEBeRG: Designing theoretically-informed implementation interventions. Implementation Sci 2006, 1:4.

16. Brehaut JC, Eva KW: Building theories of knowledge translation interventions: Use the entire menu of constructs. Implementation Sci 2012, 7:114.

17. Michie S, Johnston M, Lawton R, Parker D, Walker A: Making psychological theory useful for implementing evidence based practice: a consensus approach. Qual Saf Health Care 2005, 14:26-33.

18. Grimshaw JM, Thomas RE, MacLennan G, Fraser C, Ramsay CR, Vale L: Effectiveness and efficiency of guideline dissemination and implementation strategies. Health Technol Assess 2004, 8:1-72.

19. Colquhoun HL, Hetts JL, Law CL, Macdermid CJ, Missiuna AC: A Scoping Review of the Use of Theory in Studies of Knowledge Translation. Can J Occup Ther 2010, 77:270-279.

20. Davies P, Walker AE, Grimshaw J: A systematic review of the use of theory in the design of guideline dissemination and implementation strategies and interpretation of the results of rigorous evaluations. Implementation Sci 2010, 5:14

21. Fuhrman S: Uniting producers and consumers: Challenges in creating and utilizing educational research and development. In Education research and reform: An international perspective. Edited by Tomlinson TM, Tuijnman AC. Washington, D.C: U.S. Department of Education; 1994:133-147.

22. Hutchinson J, Huberman M: Knowledge dissemination and utilization in science and mathematics education: A literature review. Washington, D.C: National Science Foundation; 1993.

23. Vygotsky L: Mind in society: The development of higher psychological processes. Cambridge, MA: Harvard University Press; 1978.

24. Driscoll MP: Psychology of Learning for Instruction. Needham, MA: Allyn and Bacon; 1994.

25. Gredler EM: Learning and instruction. Theory into practice. New Jersey: Merrill; 1997.

26. Savery JR, Duffy TM: Problem based learning: An instructional model and its constructivist framework. Educ Tech 1995, 35:31-38.

27. Slavin RE: Educational Psychology: Theory and Practice. 4th edition. Boston, USA: Allyn and Bacon; 1994.

28. Steffe LP, Gale JE: Constructivism in education. Hillside, NJ: Laurence Erlbaum; 1995.

29. Festinger L: A Theory of Cognitive Dissonance. Stanford, CA: Stanford University Press; 1957.

30. von Glaserfeld E: A constructivist approach to teaching. In Constructivism in education. Edited by Steffe LP, Gale JE. Hillside, NJ: Lawrence Erlbraum Associates; 1995:3-15.

31. Thomas A, Saroyan A, Dauphinee WD: Evidence-based practice: a review of theoretical assumptions and effectiveness of teaching and assessment interventions in health professions. Adv Health Sci Educ Theory Pract 2011, 16:253-276

32. Graham ID, Logan J, Harrison MB, Straus SE, Tetroe J, Caswell W: Lost in knowledge translation: Time for a map? J Contin Educ Health Prof 2006 26:13-24.

33. McWilliam CL, Kothari A, Ward-Griffin C, Forbes D, Leipert B: Evolving the theory and praxis of knowledge translation through social interaction: a social phenomenological study. Implementation Sci 2009, 4:26.

34. Graham ID, Tetroe J: The knowledge to action framework. In Models and frameworks for implementing evidence-based practice: Linking evidence to action. Edited by Rycroft-Malone J, Bucknell T. Oxford, UK: Wiley-Blackwell; 2010:207-222.

35. Arksey H, O'Malley L: Scoping studies: Towards a methodological framework. Int J Soc Res Methodol 2005, 8:19-32

36. McKibbon KA, Lokker C, Wilczynski NL, Ciliska D, Dobbins M, Davis DA: A cross-sectional study of the number and frequency of terms used to refer to knowledge translation in a body of health literature in 2006: A Tower of Babel? Implementation Sci 2010, 5:16.

37. Menon A, Korner-Bitensky N, Kastner M, McKibbon KA, Straus S: Strategiese for rehabilitation professionals to move evidence-based knowledge into practice: a systematic review. J Rehab Med 2009, 41:1024-1032

38. Caley LM, Riemer S, Weinstein HS: Results of a nurse-led workshop designed to prevent fetal alcohol spectrum disorder. Public Health Nurs 2010, 27:232-239

39. Daley BJ: Learning and professional practice: A study of four professions. Adult Educ Q 2001, 52:39-54.

40. Hunter JL: Applying constructivism to nursing education in cultural competence: a course that bears repeating. J Transcult Nurs 2008, 19:354-362.

41. Rogal SMM, Snider PD: Rethinking the lecture: the application of problem based learning methods to atypical contexts. Nurse Educ Pract 2008, 8:213-219.

42. Smith CM: Comparison of Web-based instructional design strategies in a pain management program for nursing professional development. Buffalo: State University of New York; 2007.

43. Tilleczek K, Pong R, Caty S: Innovations and issues in the delivery of continuing education to nurse practitioners in rural and northern communities. Can J Nurs Res 2005, 37:146-162.

44. Abad-Corpa E, Meseguer-Liza C, Martinez-Corbalan JT, Zarate-Riscal L, Caravaca-Hernandez A, Paredes-Sidrach de Cardona A, Carrillo-Alcaraz A, Delgado-Hito P, Cabrero-Garcia J: Effectiveness of the implementation of an evidence-based nursing model using participatory action research in oncohematology: research protocol. J Adv Nurs 2010, 66:1845-1851.

45. Cronin M, Connolly C: Exploring the use of experiential learning workshops and reflective practice within professional practice development for post-graduate health promotion students. Health Educ $J$ 2007, 66(3):286-303.

46. Adler RH, von Uexkull T, Herrmann JM: The two faces of medical evidence. Swiss Med Wkly 2002, 132:397-400.

47. Appleton JV, King L: Journeying from the philosophical contemplation of constructivism to the methodological pragmatics of health services research. J Adv Nurs 2002, 40:641-648.

48. Labonte R, Robertson A: Delivering the goods, showing our stuff: the case for a constructivist paradigm for health promotion research and practice. Health Educ Q 1996, 23:431-447.

49. Plack MM: Human Nature and Research Paradigms: Theory Meets Physical Therapy Practice. Qualitative Report 2005, 10:223-245.

50. Miller KE, Kulkarni M, Kushner H: Beyond trauma-focused psychiatric epidemiology: Bridging research and practice with war-affected populations. Am J Orthopsychiatry 2006, 76(4):409-422.

51. Wilson HJ: The myth of objectivity: is medicine moving towards a social constructivist medical paradigm? Fam Pract 2000, 17:203-209.

52. Hoshmand LT, Polkinghorne DE: Redefining the science-practice relationship and professional training. Am Psychol 1992, 47:55-66.

53. Fonville AGM: How nurse executives acquire and use ethics knowledge in management decision-making. Columbia University Teachers College; 2002.

54. Carr SM: Knowing nursing - The challenge of articulating knowing in practice. Nurse Educ Pract 2005, 5:333-339.

55. Field DE: Moving from novice to expert - the value of learning in clinical practice: a literature review. Nurse Educ Today 2004, 24:560-565.

56. Higgs J, Titchen A: The nature, generation and verification of knowledge. Physiotherapy 1995, 81(9):521-530.

57. Kinsella EA: Professional knowledge and the epistemology of reflective practice. Nurs Philos 2010, 11:3-14

58. McGuckin C, Burke D: Best evidence medical education in psychiatry training. Australas Psychiatr 2002, 10(4):348-352.

59. Lipman T, Murtagh MJ, Thomson R: How research-conscious GPs make decisions about anticoagulation in patients with atrial fibrillation: a qualitative study. Fam Pract 2004, 21:290-298.

60. Schluter J, Seaton P, Chaboyer W: Understanding nursing scope of practice: A qualitative study. Int J Nurs Stud 2011, 48:1211-1222. 
61. Fairweather C, Gardner G: Specialist nurse: an investigation of common and distinct aspects of practice. Collegian 2000, 7:26-33.

62. Fagan D: Children. Child abuse and neglect: the knowledge and practice of the A and E nurse. Accid Emerg Nurs 1998, 6:30-35.

63. Greenslade MV, Elliott B, Mandville-Anstey SA: Same-day breast cancer surgery: a qualitative study of women's lived experiences. Oncol Nurs Forum 2010, 37:E92-97.

64. Rogers D, Lingard L, Boehler ML, Espin S, Klingensmith M, Mellinger JD, Schindler N: Teaching operating room conflict management to surgeons: clarifying the optimal approach. Med Educ 2011, 45:939-945.

65. Greenhalgh T, Russell J: Promoting the skills of knowledge translation in an online master of science course in primary health care. J Contin Educ Health Prof 2006, 26:100-108.

66. French SD, Green SE, O'Connor DA, McKenzie JE, Francis JJ, Michie S: Developing theory-informed behavior change interventions to implement evidence into practice: a systematic appraoch using the Theoretical Domains Framework. Implementation Sci 2012, 7:38.

67. Craik J, Rappolt S: Enhancing research utilization capacity through multifaceted professional development. Am J Occup Ther 2006, 60:155-164.

68. Moulding NT, Silagy CA, Weller DP: A framework for effective management of change in clinical practice: dissemination and implementation of clinical practice guidelines. Qual Health Care 1999, 8:177-183.

69. Estabrooks CA: The conceptual structure of research utilization. Res Nurs Health 1999, 22:203-216.

70. Rycroft-Malone J, Harvey GS, Kiston A, McCormack B, Titchen A: An exploration of the factors that influence the implementation of evidence into practice. J Clin Nurs 2004, 13:913-924.

71. Grimshaw J, Eccles M, Walker A, Thomas RE: Changing physicians' behavior: What works and thoughts on getting more things to work. J Contin Educ Health Prof 2002, 22:237-243.

72. Lavis JN, Robertson D, Woodside JM, McLeod CB, Abelson J: How can research organizations more effectively transfer research knowledge to decision makers? Milbank Q 2003, 81:221-248.

73. Oborn E, Barrett M, Racko G: Knowledge translation in health care: A review of the literature. In Cambridge Judge Business School Working Paper Series. 2010.

74. Rycroft-Malone J: Getting evidence into practice: the meaning of 'context'. J Adv Nurs 2002, 38:94-104.

75. Estabrooks CA, Thompson DS, Lovely JJ, Hofmeyer A: A guide to knowledge translation theory. J Contin Educ Health Prof 2006, 26:25-36.

76. Felton B: Innovation and Implementation in Mental Health Services for Homeless Adults: A Case Study. Community Ment Health J 2003, 39:309-322.

77. Holtstander L: Ways of knowing hope: Carper's fundamental patterns as a guide for hope research with bereaved palliative caregivers. Nurs Outlook 2008, 56:25-30.

78. Lyddon W, Yowell DR, Hermans HJM: The self-confrontation method: Theory, research, and practical utility. Counsell Psychol Q 2006, 19:27-43.

79. McWilliam CL, Kothari A, Ward-Griffin C, Forbes D, Leipert B: Evolving the theory and praxis of knowledge translation through social interaction: a social phenomenological study. Implementation Sci 2009, 4:26. doi:10.1186/1748-5908-4-26.

80. Neimeyer RA: Social Constructionism in the Counselling Context. Counsell Psychol Q 1998, 11:135-149.

81. Rolloff M: A Constructivism Model for Teaching Evidence-Based Practice Nurs Educ Perspect 2010, 31:291-293.

82. Varpio L, Schryer CF, Lehoux P, Lingard L: Working Off the Record: Physicians' and Nurses' Transformations of Electronic Patient RecordBased Patient Information. Acad Med 2006, 81:S35-S39.

83. Craig P, Dieppe P, Macintyre S, Michie S, Nazareth I, Petticrew M: Developing and evaluating complex interventions: the new Medical Research Council guidance. BMJ 2008, 337:a1655.
84. Lynch M: Towards a constructivist genealogy of social constructivism. In The Politics of Constructionism. Edited by Velody I, Williams R. London: SAGE Ppublications; 1998:13-32.

85. Burr V: Social Constructionism: An introduction. New York: Routledge; 2003.

86. Crotty M: The foundations of social research: Meaning and perspective in the research process. St Leonards, NSW: Allen and Unwin; 1998.

doi:10.1186/1748-5908-9-54

Cite this article as: Thomas et al:: Applications of social constructivist learning theories in knowledge translation for healthcare professionals: a scoping review. Implementation Science 2014 9:54.

\section{Submit your next manuscript to BioMed Central and take full advantage of:}

- Convenient online submission

- Thorough peer review

- No space constraints or color figure charges

- Immediate publication on acceptance

- Inclusion in PubMed, CAS, Scopus and Google Scholar

- Research which is freely available for redistribution

Submit your manuscript at www.biomedcentral.com/submit

C BioMed Central 